\title{
Die Krankenhausbudgets 2016 und 2017 im Vergleich
}

\author{
Carina Mostert, Jörg Friedrich und Gregor Leclerque \\ (c) Der/die Autor(en) 2019 \\ J. Klauber et al. (Hrsg.), Krankenhaus-Report 2019 \\ https://doi.org/10.1007/978-3-662-58225-1_16
}

\section{Zusammenfassung}

Der Beitrag untersucht die Veränderungen in den jährlich zu vereinbarenden Budgets der Jahre 2016 und 2017 auf Basis 1.230 somatischer Krankenhäuser. Deren Budgets sind ausgleichsbereinigt um 3,0 \% gestiegen, was einem Mittelzuwachs von etwa 1,8 Mrd. Euro entspricht. Diese ermittelte Budgetsteigerungsrate im ersten Umsetzungsjahr des Krankenhausstrukturgesetzes ist die niedrigste seit 2011. Dabei liegt der ausgleichsbereinigte Preiseffekt von 2,2 \% im Mittel der Vorjahre. Ursächlich für die niedrige Budgetveränderungsrate ist vielmehr eine vereinbarte Mengenentwicklung von nur 0,9\% nach einem Rekordwert im Vorjahr, die sich durch nahezu alle Leistungsbereiche zieht. Für eine Bewertung, inwieweit dies auch eine unmittelbare Folge der geänderten Budgetmechanismen für die Finanzierung von Mehrmengen ist oder gar eine Trendumkehr in der Mengenentwicklung in der stationären Versorgung eingeleitet hat, ist es noch zu früh. Fakt ist aber, dass die neuen, komplexen und streitbefangenen Detailregelungen zu deutlich späteren Verhandlungen und Umsetzungszeitpunkten geführt haben. Erstmals seit 2009 wurde weniger als die Hälfte des Gesamtbudgetvolumens innerhalb des Budgetjahrs auch umgesetzt mit den entsprechenden Konsequenzen für die Planungs- und Kalkulationssicherheit der Krankenhäuser und Kostenträger.

This article analyses the changes in the annually agreed budgets for 2016 and 2017 on the basis of 1230 somatic hospitals. The budgets of these hospitals increased by $3.0 \%$ after adjustment, which corresponds to an increase in funds of around 1.8 billion euros. This budget increase rate in the first year of implementation of the Hospital Structure Act is the lowest since 2011. The adjusted price effect of $2.2 \%$, however, is in line with the average of previous years; the reason for the low rate of budget change is rather an agreed volume development of only $0.9 \%$ after a record value in the previous year, which affects almost all service areas. It is still too early to assess to what extent this is also a direct consequence of the changed budget mechanisms for the financing of additional volumes or whether it has even initiated a trend reversal in the volume development in inpatient care. Nevertheless, it is a fact that the new, complex and controversial regulations have led to much later negotiations and implementation dates. For the first time since 2009, less than half of the total budget volume was implemented within the budget year, with the corresponding consequences for the planning and calculation security of hospitals and payers. 


\subsection{Einführung}

In diesem Beitrag werden die Veränderungen der jährlich zu vereinbarenden Budgets somatischer Krankenhäuser zwischen den Jahren 2016 und 2017 analysiert. Die Darstellung basiert auf den vorliegenden Unterlagen nach der amtlichen Aufstellungen der Entgelte und Budgetberechnung (AEB) aus 1.230 Kliniken. Es werden nur Einrichtungen betrachtet, zu denen für beide Jahre Budgetvereinbarungen vorliegen und die über den beobachteten Zeitraum hinweg als eigenständige Leistungserbringer am Markt präsent waren. Einrichtungen, die 2017 durch Schließungen aus dem Markt ausgeschieden oder durch Fusionen in anderen Häusern aufgegangen sind, bleiben unberücksichtigt. Die Grundgesamtheit repräsentiert 79,5\% der bundesweiten Leistungsmenge (DRG-Casemixsumme), wie sie im Rahmen der Vereinbarung der Landesbasisfallwerte (LBFW) 2017 festgelegt worden ist.

Der Beitrag beginnt in Abschn. 16.2 mit der Darstellung der allgemeinen Budgetentwicklung und der Preis- und Mengenfaktoren. In $>$ Abschn. 16.3 werden die Preis- und in $>$ Abschn. 16.4 die Leistungsentwicklungen im DRG-Bereich und für $\mathrm{Zu}$ satzentgelte vertiefend analysiert. Abschließend thematisiert $>$ Abschn. 16.5 die Wirkung der Budgetergebnisse im Zusammenhang mit den Umsetzungszeitpunkten.

\subsection{Allgemeine Budgetentwicklung}

Das vereinbarte Gesamtbudget, das die Beträge für DRGs, sonstige Entgelte nach $₫ 6$ Krankenhausentgeltgesetz (KHEntgG) sowie $\mathrm{Zu}$ - und Abschläge umfasst, steigt für die hier betrachteten Einrichtungen zwischen 2016 und 2017 um 3,0 \% auf 59,6 Mrd. Euro an. Damit liegt die niedrigste Veränderungsrate seit 2011 vor. Maßgeblich für diesen vergleichsweise moderaten Budgetanstieg im Jahr 2017 ist das vereinbarte Mengenwachstum von 0,9\%, das in den Vorjahren und besonders im Jahr 2016 höher ausfiel. Die Preiskomponente i. H. v. 2,2 \% liegt dagegen leicht über dem Durchschnittswert der Vorjahre von 2,1\% (•Tab. 16.1).

Von 2016 nach 2017 macht die Preisentwicklung über $70 \%$ der Gesamtbudgetveränderung aus.
- Tab. 16.1 Jährliche Budgetveränderung und der Einfluss von Menge und Preis

\begin{tabular}{|l|l|l|l|}
\hline Jahr & Gesamtbudget & Menge & Preis \\
\hline 2009 & $7,0 \%$ & $3,6 \%$ & $3,2 \%$ \\
\hline 2010 & $5,8 \%$ & $3,3 \%$ & $2,5 \%$ \\
\hline 2011 & $2,9 \%$ & $2,9 \%$ & $0,0 \%$ \\
\hline 2012 & $4,0 \%$ & $2,7 \%$ & $1,3 \%$ \\
\hline 2013 & $4,5 \%$ & $1,6 \%$ & $2,9 \%$ \\
\hline 2014 & $4,4 \%$ & $1,5 \%$ & $2,8 \%$ \\
\hline 2015 & $3,6 \%$ & $1,9 \%$ & $1,8 \%$ \\
\hline 2016 & $5,0 \%$ & $2,7 \%$ & $2,3 \%$ \\
\hline 2017 & $3,0 \%$ & $0,9 \%$ & $2,2 \%$ \\
\hline & & Krankenhaus-Report 2019 \\
\hline
\end{tabular}

Drei Viertel der Mengenentwicklung sind auf einen Fallzahlanstieg zurückzuführen, nur ein Viertel ist durch eine Veränderung der Leistungsstruktur bedingt (• Abb. 16.1).

Das DRG-Budget ist aufgrund des großen Anteils am Gesamtbudget (96,6\%) mit einem Anstieg um 1,6 Mrd. Euro beziehungsweise 2,9\% nach wie vor Haupttreiber der Entwicklung. Die Gesamtbeträge für Zusatzentgelte $(+6,2 \%)$ weisen deutlich stärkere, die Sonstigen Entgelte (+2,1\%) moderatere Veränderungsraten auf. Beim Gesamtbudget kaum ins Gewicht fällt die Entwicklung der $\mathrm{Zu}$ - und $\mathrm{Ab}$ schläge, obwohl hier eine Veränderung von $86,2 \% \mathrm{zu}$ verzeichnen ist (•Tab. 16.2). Details zu den Veränderungen bei Zusatzentgelten und $\mathrm{Zu}$ - und Abschlägen finden sich in $>$ Abschn. 16.3 und $>$ Abschn. 16.4.

Wird das vereinbarte Budget überschritten, werden Rückzahlungen der Krankenhäuser fällig. Kommt es hingegen zu Budgetunterschreitungen, resultieren Nachzahlungen der Krankenkassen. Diese Ausgleiche werden mit den Budgets der Folgejahre verrechnet. Während die Nach- und Rückzahlungen im Jahr 2016 nahezu ausgeglichen waren, sind sie im Jahr 2017 mit 24,1 Mio. Euro leicht im Plus.

\subsection{Vereinbarte Preisentwicklung}

Das Vergütungsniveau stationärer Leistungen im somatischen Bereich wird wie oben beschrieben hauptsächlich von der Preisentwicklung für DRG- 


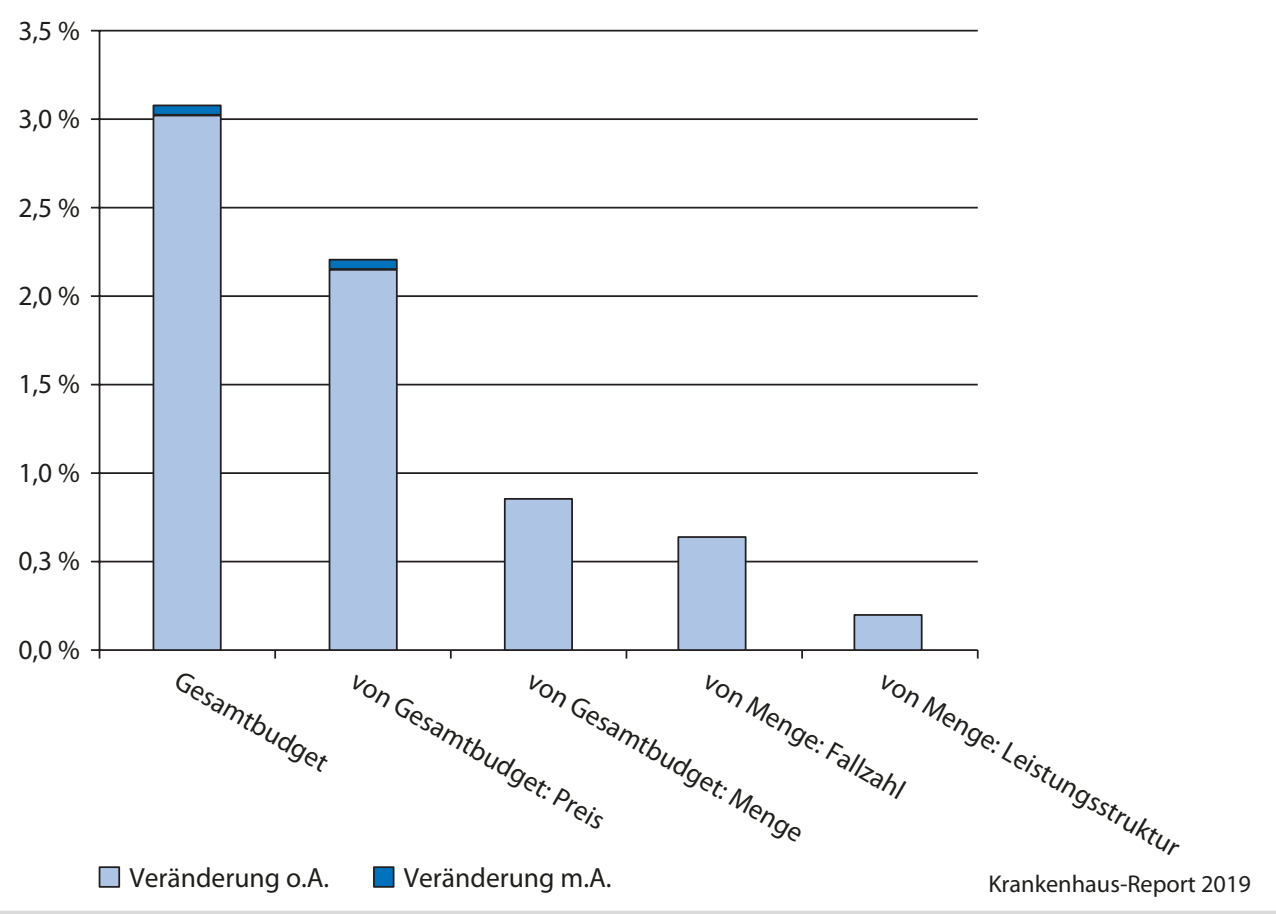

- Abb. 16.1 Einflussfaktoren der vereinbarten Budgetentwicklung 2017 ( $n=1.230$ Krankenhäuser)

- Tab. 16.2 Vereinbarte Budgets 2016 und 2017 (in Mio. Euro)

\begin{tabular}{|l|r|r|r|}
\hline & \multicolumn{1}{|c|}{2016} & \multicolumn{1}{|c|}{2017} & $\begin{array}{l}\text { Verän- } \\
\text { derung }\end{array}$ \\
\hline DRG-Budget & $55.971,0$ & $57.577,9$ & $2,9 \%$ \\
\hline Zusatzentgelte & $2.051,8$ & $2.179,5$ & $6,2 \%$ \\
\hline Sonstige Entgelte & $1.754,9$ & $1.791,3$ & $2,1 \%$ \\
\hline $\begin{array}{l}\text { Zu- und Abschläge } \\
\text { (ohne Ausbildung) }\end{array}$ & 130,8 & 243,5 & $86,2 \%$ \\
\hline Gesamtbudget & $57.856,7$ & $59.612,6$ & $3,0 \%$ \\
\hline Ausgleiche & $-4,0$ & 24,2 & $-706,2 \%$ \\
\hline Gesamtbudget mA & $57.852,7$ & $59.636,8$ & $3,1 \%$ \\
\hline $\mathrm{n}=1.230$ Krankenhäuser & & \\
\hline
\end{tabular}

Krankenhaus-Report 2019
Leistungen bestimmt. Die sogenannten Sonstigen Entgelte nach $₫ 6$ KHEntgG, deren Preise hausindividuell $\mathrm{zu}$ vereinbaren sind, spielen für die Gesamtentwicklung auf Bundesebene eine nachgeordnete Rolle und werden daher im Weiteren nicht näher untersucht.

Die DRG-Preiskomponente setzt sich maßgeblich aus den Komponenten Basisfallwert, $\mathrm{Zu}$ - und Abschläge sowie periodenfremde Ausgleiche für Budgetabweichungen in Vorjahren zusammen. Der in den Budgetverhandlungen auf Krankenhausebene verwendete Basisfallwert für die hier untersuchten Einrichtungen betrug 2016 im Mittel 3.289 Euro und steigt im Folgejahr um 2,02 \% auf 3.356 Euro an. Unter Berücksichtigung der $\mathrm{Zu}$ - und Abschläge resultiert eine Veränderung um 2,15\%. Unter Berücksichtigung der Ausgleichzahlungen für Vorperioden liegt die Preissteigerung leicht verändert bei 2,20 \%. Im Folgenden werden die bedeutenden Einflussgrößen im Detail dargestellt sowie deren Einfluss auf die Gesamtentwicklung analysiert. 


\section{- Obergrenze für die Preisentwicklung der Landesbasisfallwerte (Grundlohnrate/ Orientierungswert/Veränderungswert)}

Mit Einführung der LBFW im Jahr 2005 galt die Veränderungsrate nach $₫ 71$ SGB V Abs. 3 (Grundlohnrate) als Obergrenze für vereinbarte Preisveränderungen. Die Grundlohnrate spiegelt die Einnahmenentwicklung der gesetzlichen Krankenkassen wider. Seit 2013 soll sich die Obergrenze stärker an den Kosten der Krankenhäuser orientieren. Dazu berechnet das Statistische Bundesamt mit dem sogenannten Orientierungswert die Kostenentwicklung der Inputfaktoren für Krankenhausleistungen. Diese entspricht einer krankenhausspezifischen Inflationsrate. Die aktuell gültige Regelung für die Obergrenze der Preisentwicklung wurde mit dem Beitragsschuldengesetz 2014 eingeführt. Ob sich die Preise kosten- oder einnahmeorientiert entwickeln sollen, hängt seitdem davon $\mathrm{ab}$, ob der Orientierungswert oder die Grundlohnrate höher ist. Der höhere Wert gilt als Obergrenze.

Der vom Statistischen Bundesamt veröffentlichte Orientierungswert für das Jahr 2017 liegt mit $1,54 \%$ fast einen Prozentpunkt unterhalb der veröffentlichten Veränderungsrate nach $\$ 71$ SGB V Abs. 3 in Höhe von 2,5 \%. Daher galt 2017 wiederholt die Grundlohnsumme als Obergrenze für die Veränderung der LBFW. Im gewichteten Mittel stiegen die LBFW mit Ausgleichen von 3.294 Euro im Jahr 2016 um 2,04 \% auf 3.358 Euro im Jahr 2017. Somit blieb die vereinbarte Preisveränderung mit 0,44 Prozentpunkten deutlich unterhalb der geltenden Obergrenze.

\section{- Tariferhöhungsrate 2017}

Im Gesetz zur Beseitigung sozialer Überforderung bei Beitragsschulden in der Krankenversicherung wurde eine einmalige anteilige Refinanzierung der Tarifsteigerungen für das Jahr 2013 vorgesehen (Mostert et al. 2015). Mit dem Krankenhausstrukturgesetz (KHSG) wurde in Form der Tariferhöhungsrate eine dauerhafte Tarifrefinanzierung eingeführt. Ab 2016 vereinbaren die DKG, die PKV und der GKV-Spitzenverband jährlich die Differenz zwischen der Obergrenze und den Tariflohnsteigerungen und weisen diese als Erhöhungsrate aus. 2016 war dieser Wert nicht zu vereinbaren, da die
Tariferhöhungen unterhalb des Veränderungswerts von 2,95\% lagen. Für 2017 vereinbarten die Vertragsparteien eine Tariferhöhungsrate von $0,48 \%$, was eine anteilige Erhöhungsrate im DRG-Bereich von $0,16 \%$ nach sich zog. In ihrer gemeinsamen Empfehlung zur Umsetzung dieser Erhöhungsrate vom Ende September 2017 einigten sich Vertragsparteien darauf, diese Erhöhung erst in den LBFW des Jahres 2018 zu berücksichtigen. Somit hatte die Tariferhöhungsrate im laufenden Jahr 2017 keine budgeterhöhende Wirkung.

\section{- Hygienesonderprogramm}

Ebenfalls mit dem Beitragsschuldengesetz wurde die Förderung der Krankenhaushygiene im KHEntgG eingeführt. Ursprünglich sollten Krankenhäuser zusätzliche Mittel für die Neueinstellung und Weiterbildung von ärztlichem und pflegerischem Hygienepersonal für die Jahre 2013 bis 2016 erhalten. Mit dem Krankenhausstrukturgesetz von 2016 wurde das Programm um weitere drei Jahre bis 2019 verlängert.

Für das Jahr 2016 beträgt das vereinbarte Budgetvolumen in den hier untersuchten Krankenhäusern für das Hygienesonderprogramm 72,8 Mio. Euro und steigt 2017 um 5,1\% auf 76,5 Mio. Euro.

\section{- Pflegestellenförderprogramm}

Mit dem Krankenhausstrukturgesetz (KHSG) wurde das zweite Pflegestellenförderprogramm für die Budgetjahre 2016 bis 2018 aufgelegt. In diesem drei Jahren können die Verhandlungspartner jährlich bis zu 0,15\% des Krankenhausbudgets ${ }^{1}$ zusätzlich für Neueinstellungen oder Aufstockung vorhandener Teilzeitstellen im Pflegedienst vereinbaren. Dabei haben die Krankenhäuser einen Eigenanteil von $10 \%$ aufzubringen; diese Vorgabe deckt sich mit dem ersten Förderprogramm. ${ }^{2}$

1 Bezogen auf den Gesamtbetrag nach $\S 4$ Abs. 3 S. 1 KHEntgG.

2 Das erste Pflegesonderprogramm lief in den Jahren 2009 bis 2011 und beinhaltete eine Förderung der Kosten von Neueinstellungen und Stellenaufstockungen in der Krankenhauspflege von bis zu $90 \%$ durch die gesetzlichen Krankenkassen. Die Krankenhäuser hatten in diesen Jahren Anspruch auf eine Förderung in Höhe von 0,48\% ihres Budgets. 
Im Vergleich zu den 52,9 Mio. Euro aus 2016 hat sich in den hier betrachteten Krankenhäusern das vereinbarte Volumen 2017 mit 132 Mio. Euro mehr als verdoppelt. Die Auswirkungen des Pflegestellenförderprogramms auf den Preis von DRG-Leistungen summieren sich 2017 auf 6,58 Euro, im Vorjahr betrug dieser Wert noch 3,11 Euro. Bezogen auf die gesetzlich festgelegte Obergrenze für die Finanzierung des Programms i. H. v. 0,15\% der Erlösbudgets p. a. beträgt der Umsetzungsgrad des Programms ca. $63 \%$.

\section{- G-BA-Mehrkostenzuschlag}

Darüber hinaus wurden mit dem KHSG befristete Zuschläge für die Finanzierung von Mehrkosten aufgrund von Richtlinien des Gemeinsamen Bundesausschusses etabliert. Im April 2017 trat die entsprechende Vereinbarung zwischen den Vertragspartnern auf Bundesebene in $\mathrm{Kraft}^{3}$ In deren Anlage 1 wird auch die konkrete Zuschlagsfinanzierung der Qualitätssicherungs-Richtlinie Frühund Reifgeborene (QFR-RL) geregelt. Sie legt die Geltungsdauer der befristeten Zuschläge vom 05.11.2015 bis zum 31.12.2021 fest, wobei die $\mathrm{Zu}$ schlagsfinanzierung für die Jahre 2015 und 2016 in den Budgets ab 2017 erfolgt. Für das Budgetjahr 2017 beträgt die vereinbarte Budgetsumme 79,8 Mio. Euro aus 116 der insgesamt 1.230 vorliegenden Vereinbarungen. Der Effekt auf den Preis von DRG-Leistungen im Jahr 2017 beträgt entsprechend 4,65 Euro.

\section{- Mehrleistungsabschlag}

Hinsichtlich der Vergütung von vereinbarten Leistungsveränderungen bestehen seit Beginn der Konvergenzphase im Jahr 2005 unterschiedliche gesetzliche Auflagen, die in den Budgetverhandlungen zu berücksichtigen sind. Hintergrund ist, dass steigende Mengen in den meisten Leistungsbereichen c. p. zu sinkenden Durchschnittskosten führen, da sich lediglich die variablen Kosten verändern und die Fixkosten konstant bleiben.

3 „Vereinbarung gemäß $§ 9$ Abs. 1 a Nr. 1 KHEntgG zur Finanzierung von Mehrkosten aufgrund der Richtlinien des Gemeinsamen Bundesausschusses zur Qualitätssicherung (G-BA-Mehrkostenzuschlagsvereinbarung)“.
Mit dem 2012 verabschiedeten Psychiatrie-Entgeltgesetz (PsychEntgG) wurde der Mehrleistungsabschlag ab 2013 mit einer Geltung für zwei Jahren auf $25 \%$ festgelegt. Mit dem ersten Pflegestärkungsgesetz (PSG I) von 2014 wurde eine Verlängerung der Geltungsdauer auf drei Jahre geregelt. ${ }^{4}$ Mit Inkrafttreten des Krankenhausstrukturgesetzes (KHSG) am 01. Januar 2016 wurden die Regelungen zur Steuerung und Budgetberücksichtigung von Leistungsveränderungen für das Folgejahr deutlich geändert: Ab dem Jahr 2017 entfällt der Mehrleistungsabschlag für neu vereinbarte Mehrmengen und mit dem Fixkostendegressionsabschlag (FDA) wird ein neues Instrumentarium eingeführt (s. u.).

Das vereinbarte Gesamtvolumen für den Mehrleistungsabschlag 2016 inkl. der weitergeltenden Beträge aus den Vorjahren beläuft sich auf 538,9 Mio. Euro. Dies entspricht einem vereinbarten Preiseffekt von -31,67 Euro. Im Jahr 2017 beträgt dieser Effekt aufgrund der weitergeltenden Beträge aus den Vorjahren in Höhe von 382,0 Mio. Euro noch -22,27 Euro (• Abb. 16.2).

\section{- Wegfall der Berücksichtigung von Mengen- veränderung im Landesbasisfallwert/ \\ Fixkostendegressionsabschlag}

Trotz seiner auf den ersten Blick großen Ähnlichkeiten zum im gleichen Jahr entfallenden Mehrleistungsabschlag ersetzt der mit dem KHSG ab 2017 eingeführte Fixkostendegressionsabschlag (FDA) zusätzlich die bisher auf Landesebene im LBFW wirksame Mengendegression. Für den FDA ist eine Laufzeit von drei Jahren vorgesehen. Somit tritt ein befristeter krankenhausindividueller Abschlag an die Stelle einer dauerhaften Wirkung im LBFW, um die Skaleneffekte aus der Erbringung von Mehrleistungen abzubilden. Ursprünglich sollten die Vertragspartner auf Landesebene die Höhe des Regelabschlags jährlich vereinbaren, für die Jahre 2017 und 2018 wurde der FDA-Regelsatz jedoch auf $35 \%$ gesetzlich festgelegt. Dieser Satz gilt für alle Regelleistungen, die nicht unter einen Ausnahme-

4 Von den Regelungen ausgenommen sind Mehrleistungen aus DRGs mit einem Sachkostenanteil von mehr als 66,7 \% oder solche, die aus krankenhausplanerischen Maßnahmen resultieren. 


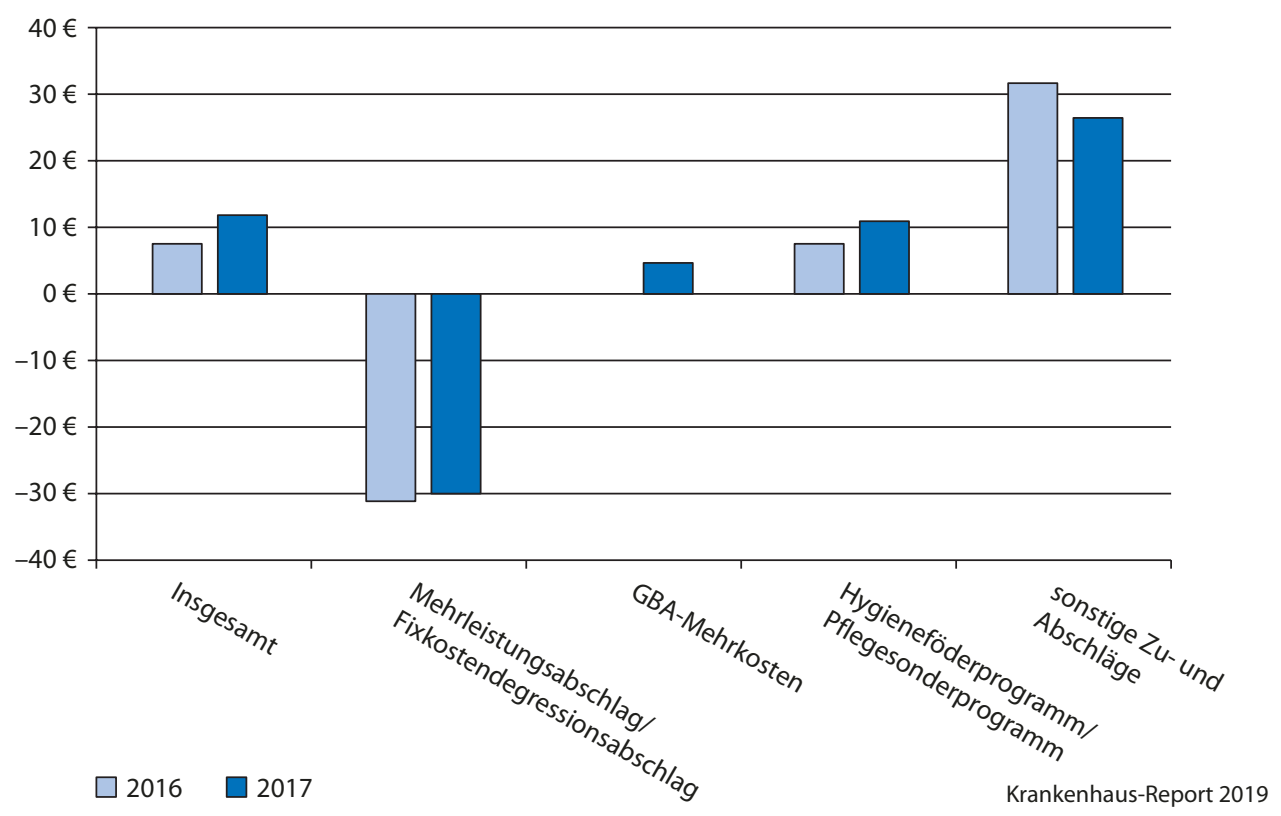

- Abb. 16.2 Preiswirkung von Zu- und Abschlägen auf den Basisfallwert 2016/2017 ( $n=1.230$ Krankenhäuser)

tatbestand ${ }^{5}$ oder eine Sonderreglung ${ }^{6}$ fallen, die den Abschlag auf die Hälfte reduziert. Ein erhöhter Abschlag von bis zu 50 \% kann für zusätzliche Leistungen mit höherer Fixkostendegression vereinbart werden oder wenn in hohem Maße wirtschaftlich begründete Mengensteigerungen vorliegen.

Die Wirkung aus dem Wegfall der Berücksichtigung von Mehrleistungen in den LBFW-Vereinbarungen 2017 kann nicht beziffert werden. Für das Budgetjahr 2017 beträgt die Summe der vereinbarten Fixkostendegressionsabschläge 139,1 Mio. Euro

5 Komplett vom FDA ausgenommen sind Mehrleistungen aus den Bereichen Transplantationen, Polytraumata, Versorgung Schwerbrandverletzter, Versorgung Frühgeborener, Leistungen für zusätzliche Versorgungsaufträge sowie Leistungen von Zentren. Ferner ausgenommen sind Leistungen mit einem Sachkostenanteil von mehr als zwei Dritteln und DRGs, deren Bewertungsrelationen im Katalog abgesenkt wurden, weil Anhaltspunkte für wirtschaftlich begründete Fallzahlsteigerungen in der Vergangenheit vorliegen.

6 Der hälftige FDA kommt für DRGs aus dem Katalog der „nicht mengenanfälliger Leistungen“ zur Anwendung. Gleiches gilt im Falle von Leistungsverlagerungen im Einzugsgebiet eines Krankenhauses. aus 309 der insgesamt 1.230 vorliegenden Vereinbarungen. Der Effekt auf den Preis von DRG-Leistungen im Jahr 2017 beträgt entsprechend -8,10 Euro.

\section{- Versorgungszuschlag/Pflegezuschlag}

Seit 2013 erhalten somatische Krankenhäuser einen sogenannten Versorgungszuschlag. Dieser wurde mit dem Beitragsschuldengesetz eingeführt, um die sogenannte „doppelte Degression“ zu neutralisieren. Der Begriff „Doppelte Degression“ bezieht sich auf die Regelung, dass vereinbarte Mehrmengen sowohl in den LBFW (bis einschließlich 2016) als auch über den Mehrleistungsabschlag auf Hausebene preisdämpfend wirkten (s. o.). Der Versorgungszuschlag wurde aber nicht so konzipiert, dass er die Summe der Mehrleistungsabschläge ausschüttet, sondern als ein fixer prozentualer Aufschlag auf DRG-Fallpauschalen. Für das Jahre 2016 betrug der Zuschlag 0,8\%. Das erste Pflegestärkungsgesetz (PSG I) regelte den Übergang des Versorgungszuschlags in den Pflegezuschlag ab 2017, obwohl mit der Abschaffung der Mengenberücksichtigung in den LBFW ab 2017 die „doppelte Degression“ nicht mehr vorlag. Das KHSG hat das 
jährliche Zuschlagsvolumen auf 500 Millionen konkretisiert. Die Fördersumme jedes einzelnen Hauses leitet sich aus dessen Anteil der Personalkosten für das Pflegepersonal an den Personalkosten für das Pflegepersonal aller allgemeinen Krankenhäuser im Bund ab. ${ }^{7}$ Somit erfolgt die Ausschüttung nicht mehr pauschal über die Gesamterlöse im DRG-Bereich (Versorgungszuschlag), sondern über den konkreteren Aufwand für Pflegepersonal (Pflegezuschlag).

Für 2016 beläuft sich der Versorgungszuschlag für die hier untersuchten Krankenhäuser auf die kalkulatorische Summe von 447,8 Mio. Euro. Das vereinbarte Volumen des Pflegezuschlags im Jahr 2017 beläuft sich auf 400,1 Mio. Euro. ${ }^{8}$ Der Preiseffekt in den Jahren 2016 und 2017 liegt bei $+26,31$ Euro beziehungsweise $+23,32$ Euro.

\subsection{Vereinbarte Leistungs- entwicklung}

Die folgenden zwei Abschnitte widmen sich der vereinbarten Leistungsentwicklung in den Bereichen DRG und Zusatzentgelte. Die wesentlichen Determinanten werden mit der Methode der Komponentenzerlegung identifiziert und quantifiziert.

\subsubsection{Leistungsveränderung im DRG-Bereich}

Die Leistungsmenge im DRG-Bereich wird über den Casemix (CM) ausgedrückt. Er ergibt sich aus Multiplikation der Komponenten Fallzahl und durchschnittlicher Fallschwere (CMI). Für einen zutreffenden Vergleich der vereinbarten Leistungs-

7 Für das Jahr 2017 handelt es sich dabei konkret um 15.530.774 Euro, wie sie als Personalkosten im Pflegedienst für allgemeine Krankenhäuser (Ifd. Nr. 19) für das Jahr 2014 in der Fachserie 12 Reihe 6.3 des Statistischen Bundesamts ausgewiesen sind. Der Anteil eines einzelnen Krankenhauses an diesem Betrag definiert den Anteil am Gesamtfördervolumen von 500 Mio. Euro.

8 Der Vergleich dieser beiden Werte steht vor dem Vorbehalt, dass die Beträge aus 2016 anders als 2017 nicht vereinbart, sondern aus den vereinbarten DRG-Budgets abgeleitet wurden. volumina zweier Jahre ist es erforderlich, die Veränderungen zwischen den jeweils gültigen DRG-Katalogen zu berücksichtigen. Die Effekte aus dem G-DRG-Katalogwechsel 2016/2017 werden im Folgenden dargelegt.

\section{- Auswirkungen aus der G-DRG-Katalog- revision 2016/2017 (Katalogeffekt)}

Die seit 2006 für den G-DRG-Katalog verwendete Normierungsmethode soll sicherstellen, dass die Anwendung eines neuen Kataloges gegenüber der Vorgängerversion auf nationaler Ebene zum gleichen CM-Volumen führt. Die jährliche Kalkulation des G-DRG-Katalogs durch das Institut für das Entgeltsystem im Krankenhaus (InEK) führt aber außer zur Neubewertung der jeweiligen Krankenhausleistungen auch zu strukturellen Änderungen des Entgeltsystems. Die Auswirkungen dieser Revisionen werden im Weiteren Katalogeffekt genannt.

Auf tiefer gegliederten Ebenen wie den Major Diagnostic Categories (MDCs) und deren Partitionen, aber auch auf Krankenhaus- oder Landesebene sind zum Teil deutliche Katalogeffekte nicht unüblich. Aus ihnen resultiert eine entsprechende Änderung der Vergütungs- und damit Budgethöhe ohne reale Leistungsveränderung. Um diese Störgröße $\mathrm{zu}$ neutralisieren, werden für alle vergleichenden Darstellungen in den folgenden Kapiteln die vereinbarten DRG-Leistungen des Jahres 2016 in den Katalog des Jahres 2017 überführt. ${ }^{9}$

Mit Überleitung der Vereinbarungen des Jahres 2016 auf den G-DRG-Katalog 2017 sinkt der CM für die hier betrachteten Einrichtungen um knapp 1.635 Bewertungsrelationen (BR), was einem neutralen Katalogeffekt gleichkommt. Die individuellen Katalogeffekte der Krankenhäuser liegen zwischen $-7,8 \%$ und $82,3 \%$. Die $20 \%$ der Häuser mit der negativsten katalogbedingten Veränderung verzeichnen einen CM-Rückgang von mehr als 0,6\%. Für $20 \%$ der Krankenhäuser erfolgt eine Aufwertung des vereinbarten CM-Volumens um mindes-

9 Die Abbildung der Vereinbarungen des Jahres 2016 nach G-DRG-Katalog 2017 erfolgt mit dem Verfahren der "Vereinbarungsgewichteten Überleitung". Dieses Verfahren gewichtet die vereinbarten Mengen des Jahres 2016 je DRG mit einer hausspezifischen Überleitungstabelle auf Basis von §301-Daten von AOK-Versicherten (Friedrich und Paschen 2005). 
tens $0,9 \%$ (•Tab. 16.3). Somit fällt die Spreizung der Katalogeffekte auf Hausebene erneut stärker aus als im Vorjahr (Mostert et al. 2018).

Auf Ebene der 25 Major Diagnostic Categories $(\mathrm{MDCs})^{10}$ sind die Veränderungen der in den vorangegangenen Jahren mengendynamischen MDC 8 (Krankheiten und Störungen am MuskelSkelett-System und Bindegewebe) am deutlichsten (- Abb. 16.3). Sie verliert aufgrund der Katalogrevision 51.500 BR, was einem Effekt von -1,63 \% entspricht. Die MDC 6 (Krankheiten und Störungen der Verdauungsorgane) wurde wie in den Jahren zuvor erneut aufgewertet. Für die hier untersuchten Einrichtungen summiert sich der Katalogeffekt in der MDC 6 auf zusätzliche $16.600 \mathrm{BR}$, was einem Effekt von $+1,06 \%$ gleichkommt.

\section{- Komponentenzerlegung der vereinbarten CM-Veränderung im DRG-Bereich}

Nach Bereinigung des Katalogeffekts ergibt sich eine vereinbarte Leistungsentwicklung von 2016 nach 2017 von ca. 116.600 CM-Punkten (• Abb. 16.4). Die Veränderungsrate liegt damit um 0,2 Prozentpunkte niedriger als ohne Berücksichtigung der Katalogveränderung. Zur detaillierten Analyse der Leistungsentwicklung im DRG-Bereich wird im Folgenden das Konzept der Komponentenzerlegung ${ }^{11}$ angewendet. Sie quantifiziert den Einfluss von Fallzahl und Fallschwere (CMI) und zerlegt die CMI-Entwicklung in weitere Teilkomponenten.

Isoliert betrachtet führt der Fallzahlanstieg zu einem Zuwachs von 85.000 CM-Punkten und

10 Die deutsche Bezeichnung für MDC lautet Hauptdiagnosegruppe. Eine Aufstellung aller MDCs findet sich in - Tab. 16.4.

11 Für die Anwendung der Komponentenzerlegung müssen zwei Bedingungen erfüllt sein: eine Produkthomogenität und eine ausgeprägte Produkthierarchie. Erstere wird dadurch gewährleistet, dass die Vereinbarungen beider Jahre über den DRG-Katalog 2017 abgebildet werden. Die zweite Bedingung ist durch die natürlichen Eigenschaften des DRG-Systems erfüllt, da es die Ebenen DRG, Basis-DRG, Partition und MDC vorsieht. Für Analysen im DRG-System hat das Konzept bereits mehrmals Anwendung gefunden, wie bspw. bei Friedrich und Günster 2006 und Fürstenberg et al. 2013. Für eine ausführliche Beschreibung weiterer theoretischer Grundlagen der Komponentenzerlegung siehe Reichelt 1988.
Tab. 16.3 Verteilung der Katalogeffekte auf Einzelhausebene

Katalogeffekt

\begin{tabular}{l|l}
\hline 1. Quintil & negativer als $-0,64 \%$ \\
\hline 2. Quintil & zwischen $-0,64 \%$ und $-0,03 \%$ \\
\hline 3. Quintil & zwischen $-0,03 \%$ und $0,32 \%$ \\
\hline 4. Quintil & zwischen $0,32 \%$ und $0,85 \%$ \\
\hline 5. Quintil & positiver als $0,85 \%$
\end{tabular}

$\mathrm{n}=1.230$ Krankenhäuser

Krankenhaus-Report 2019

macht wie im Vorjahr ca. drei Viertel der Gesamtveränderung aus. Der restliche Teil des Anstiegs resultiert aus der durchschnittlichen Fallschwere (CMI).

Dass sich der Trend hin zu kürzeren Verweildauern fortsetzt, zeigt der absenkende Einfluss $(-0,3 \%)$ der BR-Komponente. Eine Tendenz zur Vereinbarung höher bewerteter Leistungen, der sich an der positiven Strukturkomponente ablesen lässt, ist ebenfalls schon seit vielen Jahren zu beobachten. Sie fällt im Vergleich zum Vorjahr zwar um $-0,7$ Prozentpunkte schwächer aus, liegt aber mit $0,5 \%$ auf dem durchschnittlichen Niveau der Jahre 2013 bis 2015 (Mostert et al. 2018).

Verschiebungen innerhalb von Basis-DRGs (Intra-ADRG-Komponente) gehen mit einem Effekt von $0,1 \%$ ein. Der größte Teil des Struktureffekts ist auf die Inter-ADRG-Komponente zurückzuführen $(0,4 \%)$. Im hierarchischen Aufbau des DRGSystems können diese Verschiebungen zwischen verschiedenen Basis-DRGs

- innerhalb der gleichen MDC und Partition

(Intra-Partition) (+0,3\%),

- innerhalb der gleichen MDC aber unterschiedlichen Partitionen (Inter-Partition) (+0,1\%) und

- zwischen unterschiedlichen MDCs (Inter-

MDC) $(-0,1 \%)$

stattfinden.

- Tab. 16.4 zeigt die Komponenten der vereinbarten CM-Entwicklung je MDC und - Abb. 16.5 die Bedeutung der einzelnen MDCs für die GesamtCM-Veränderung von 2016 nach 2017. Die kleinste und größte prozentuale Veränderungsrate liegen bei 


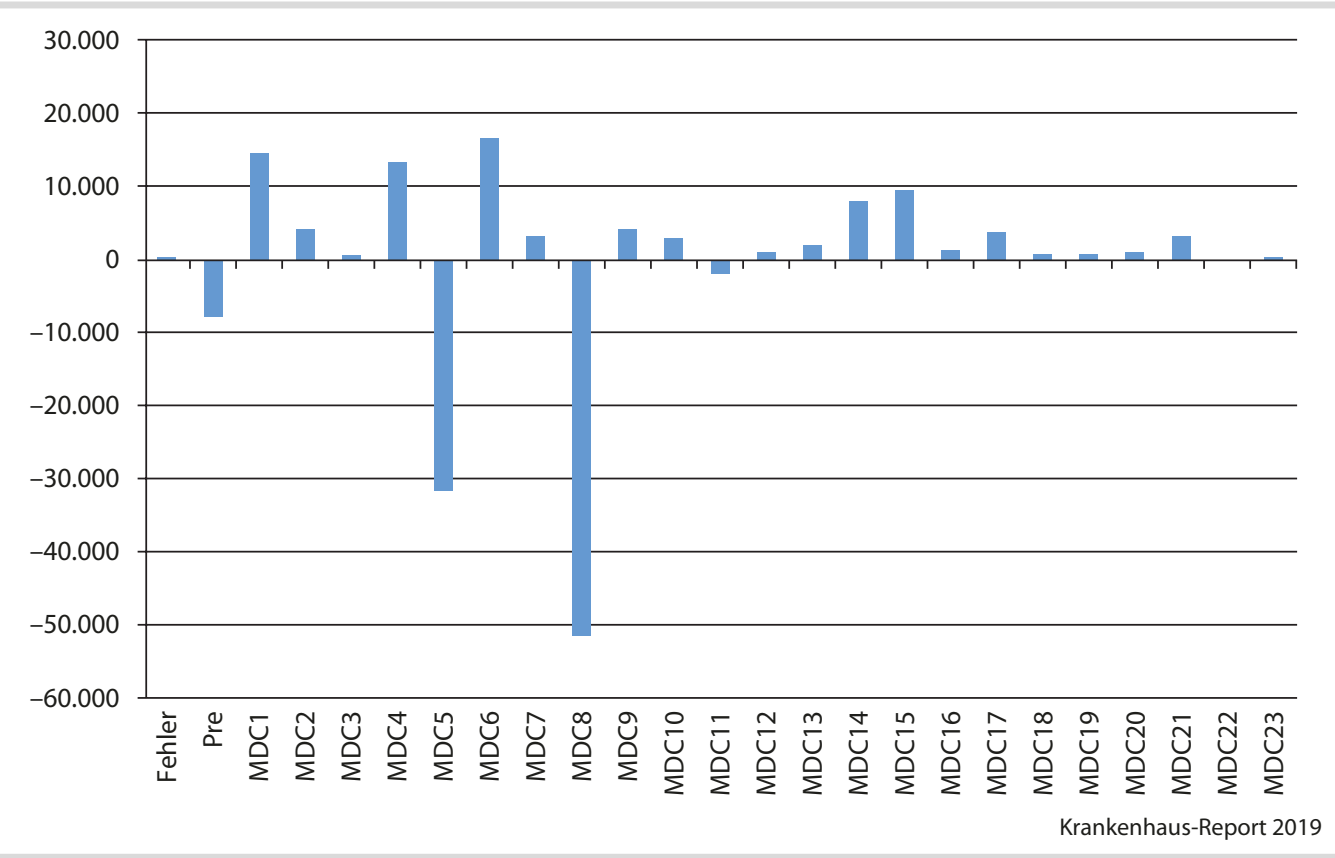

- Abb. 16.3 Absolute CM-Änderung je MDC infolge der Katalogrevision 2017 zum Vorjahr (in BR) ( $n=1.230$ Krankenhäuser)

\begin{tabular}{|r|r|rr|}
\hline Casemix & & & 116.557 \\
\hline Fälle & & & 85.099 \\
CMI & & & 31.457 \\
\hline BR & & & -49.839 \\
\hline Struktur & & & 81.296 \\
\hline Intra-ADRG & & & 23.068 \\
\hline Inter-ADRG & & & 58.229 \\
\hline Intra-Partition & & & 55.420 \\
Inter-Partition & & & 23.928 \\
Inter-MDC & & & -21.120 \\
\hline
\end{tabular}

Krankenhaus-Report 2019

- Abb. 16.4 Komponenten der vereinbarten CM-Entwicklung 2016/2017 ( $n=1.230$ Krankenhäuser)

MDCs mit kleineren CM-Volumen vor (MDC 16: $-1,9 \%$ (Blut, blutbildende Organe und Immunsystem und MDC 19: $+8,4 \%$ (Psychiatrische Krankheiten und Störungen). Von den MDCs, die mindestens $10 \%$ des gesamten-CM-Volumens aus- machen, weist die MDC 15 (Neugeborene) den deutlichsten prozentualen CM-Anstieg auf. Gemessen an der absoluten Veränderung steht sie hinter den MDCs 8 (Muskel-Skelett-System) und 4 (Atmungsorgane) an dritter Stelle. Bei fünf MDCs ist 


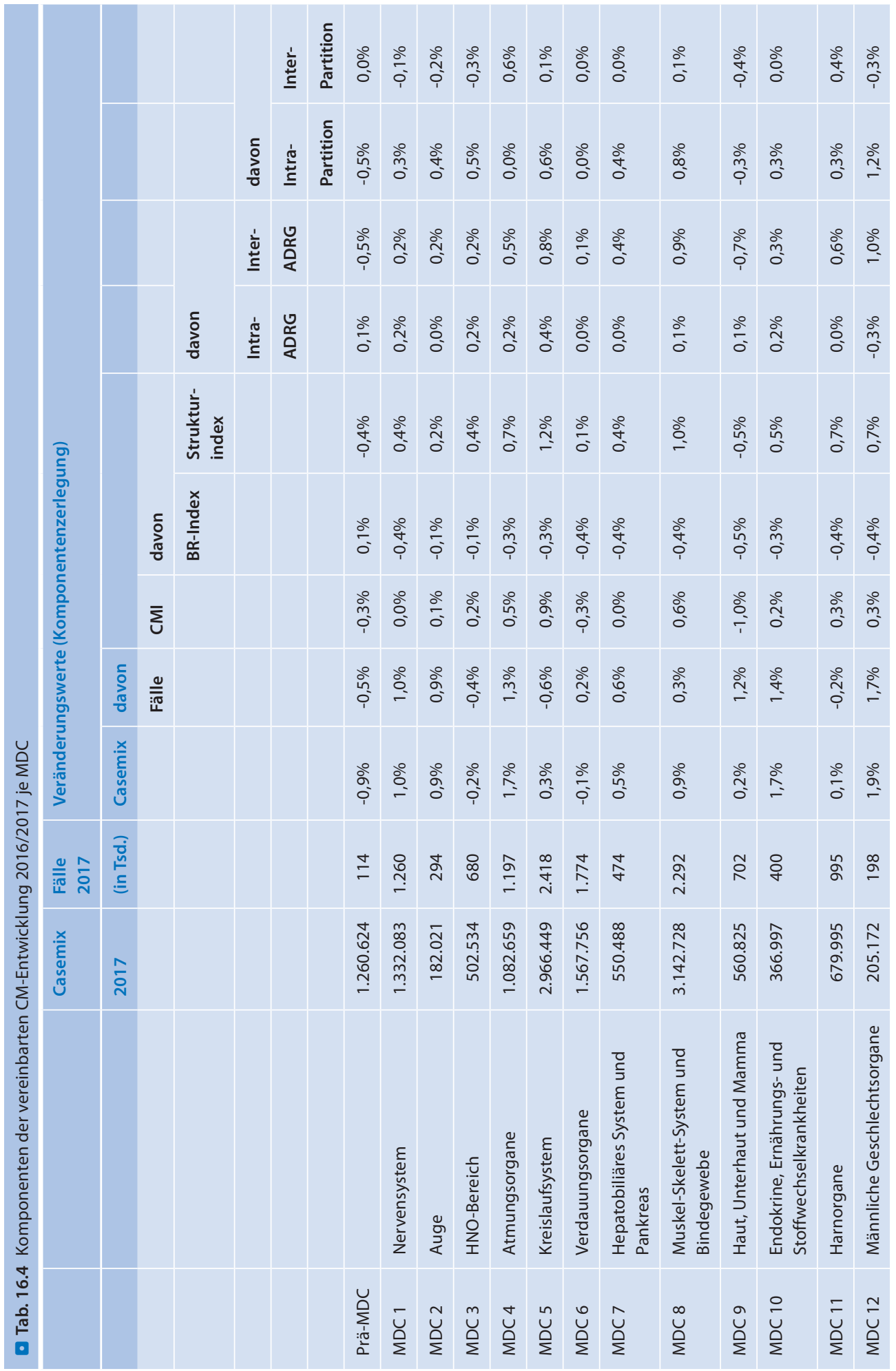




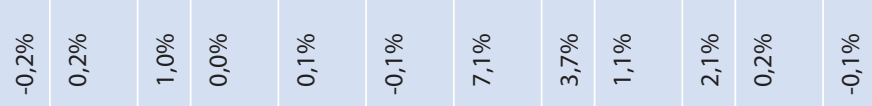

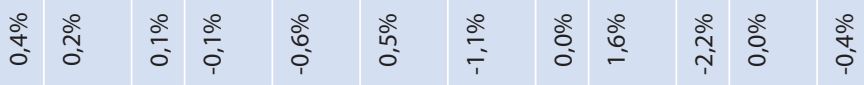

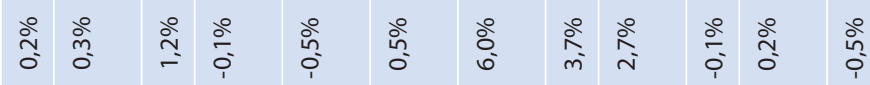

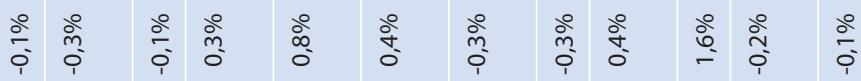

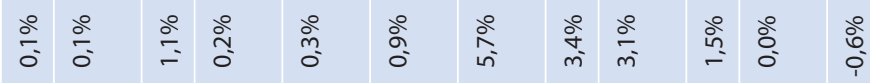

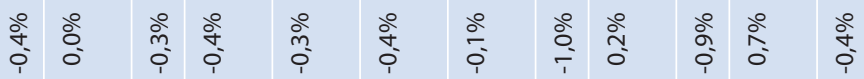

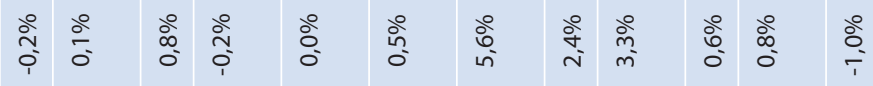

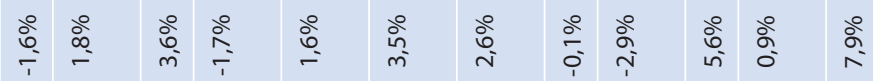

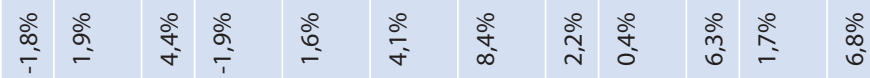

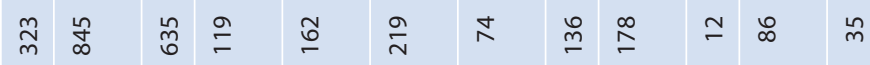

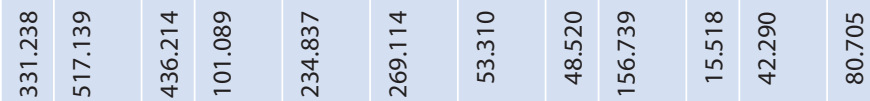

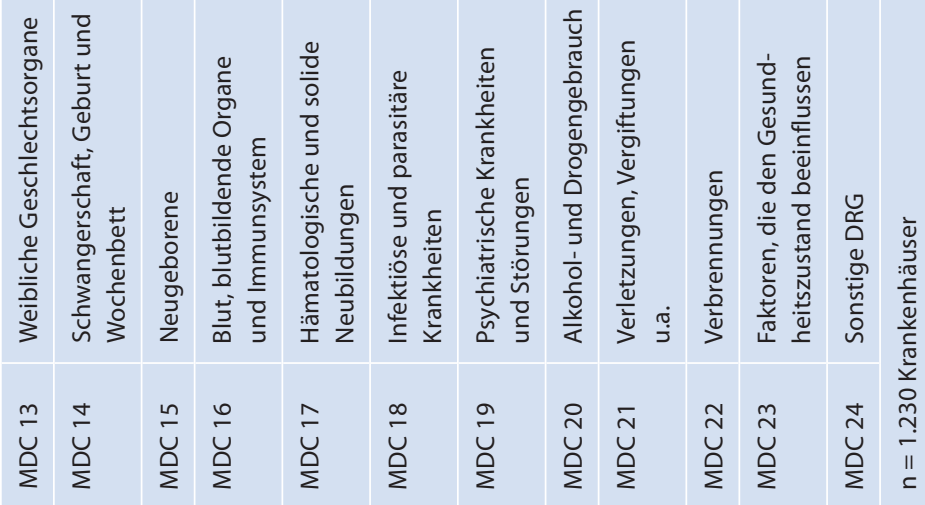




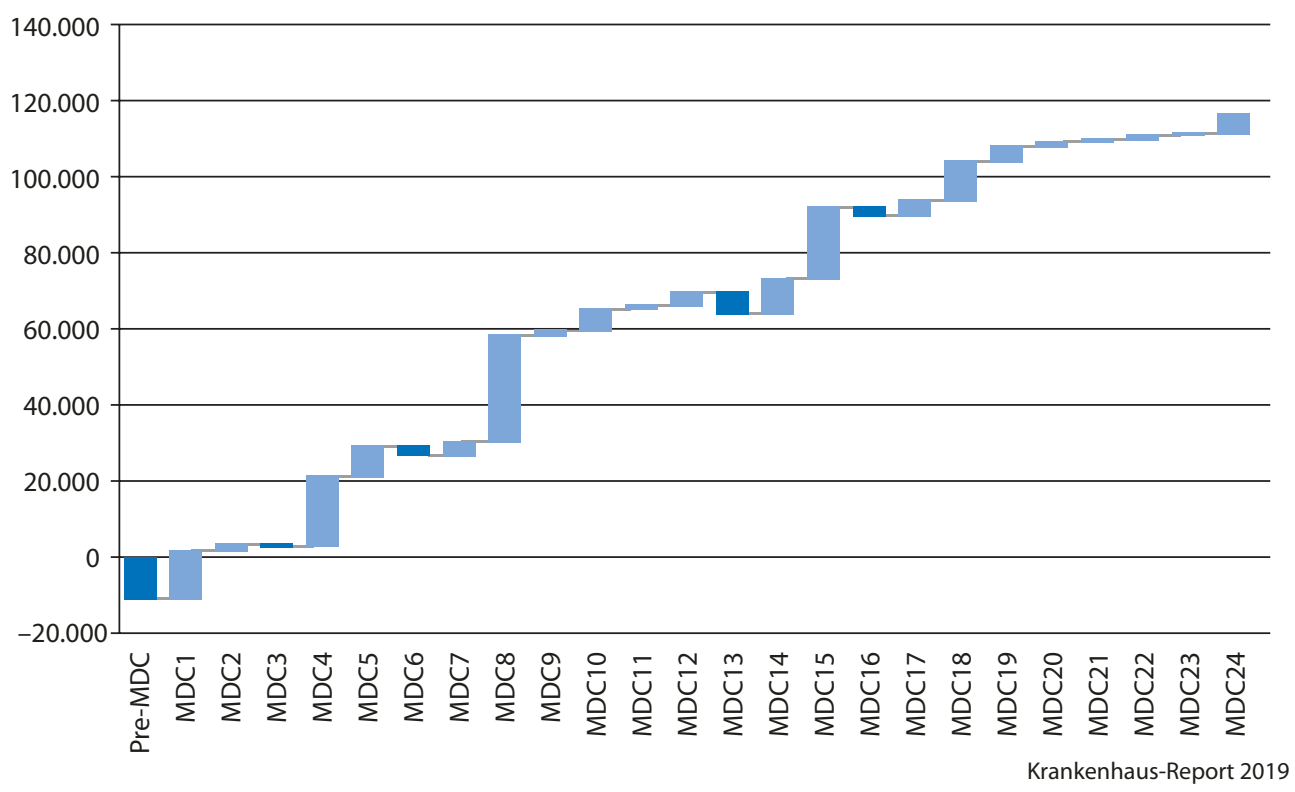

- Abb. 16.5 Absolute Veränderung des vereinbarten CM je MDC 2016 gegenüber dem Vorjahr ( $n=1.230$ Krankenhäuser)

ein CM-Rückgang zu verzeichnen, wozu mit der Pre-MDC und der MDC 5 (Verdauungsorgane) auch zwei der fünf CM-stärksten MDCs gehören.

Insgesamt machen die fünfCM-stärksten MDCs im Jahr 2017 einen Anteil von 61,5 \% des gesamten CM-Volumens aus. Bei Betrachtung ihrer jährlichen Mengenveränderungsraten ab 2013 spiegelt sich das überdurchschnittliche Wachstum im Jahr 2016 im Vergleich zu den Vorjahren wider, bevor 2017 nur noch ein vergleichsweise schwacher Zuwachs oder sogar Rückgang zu sehen ist (• Abb. 16.6). Bei allen MDCs bis auf der MDC 6 (Verdauungsorgane) übersteigt die durchschnittliche Veränderung der letzten zwei Jahre allerdings immer noch den durchschnittlichen Wert von 2013 bis 2015.

\subsubsection{Leistungsentwicklung im Zusatzentgelte-Bereich}

Zwischen 2016 und 2017 ist das Volumen der vereinbarten Zusatzentgelte für die hier betrachteten Häuser um 6,2 \% auf 2.179,5 Mio. Euro gestiegen. Ihr Anteil am Gesamtbudget beträgt 2017 in der Summe 3,7 \%.
Für einen kleineren Teil der Zusatzentgelte werden die Preise individuell mit einzelnen Krankenhäusern vereinbart, weil noch keine ausreichende, beziehungsweise ausreichend homogene Datengrundlage zur Kalkulation bundeseinheitlicher Preise durch das InEK existiert. Für den überwiegenden Teil der Zusatzentgelte ist jedoch ein bundesweit einheitlicher Preis festgelegt. Die bundesweit einheitlich bepreisten Zusatzentgelte werden in der AEB im E2-Formular erfasst, die hausindividuell vergüteten im E3.2-Formular.

Die E3.2-Zusatzentgelte machen mit einem Anteil von 32,6\% den kleineren Teil der Zusatzentgelte aus. ${ }^{12}$ Rechnerisch wächst ihr Budget mit $28,1 \%$ sehr stark an. Dies ist jedoch durch den Umstand bedingt, dass sechs umsatzstarke Arzneimittel aus dem E2- in den E3.2-Bereich gewechselt sind. Hintergrund ist der Ablauf der entsprechenden Patente für die Originalpräparate (InEK 2016, S. 144f). Auf die bundesweit einheitlich vergüteten Zusatzentgelte entfällt ein Budgetvolumen von

$12 \mathrm{Zu}$ dieser Gruppe zählen auch Zusatzentgelte für Neue Untersuchungs- und Behandlungsmethoden (NUB) und hochspezialisierte Leistungen nach § 6 Abs. 2a KHEntgG. 


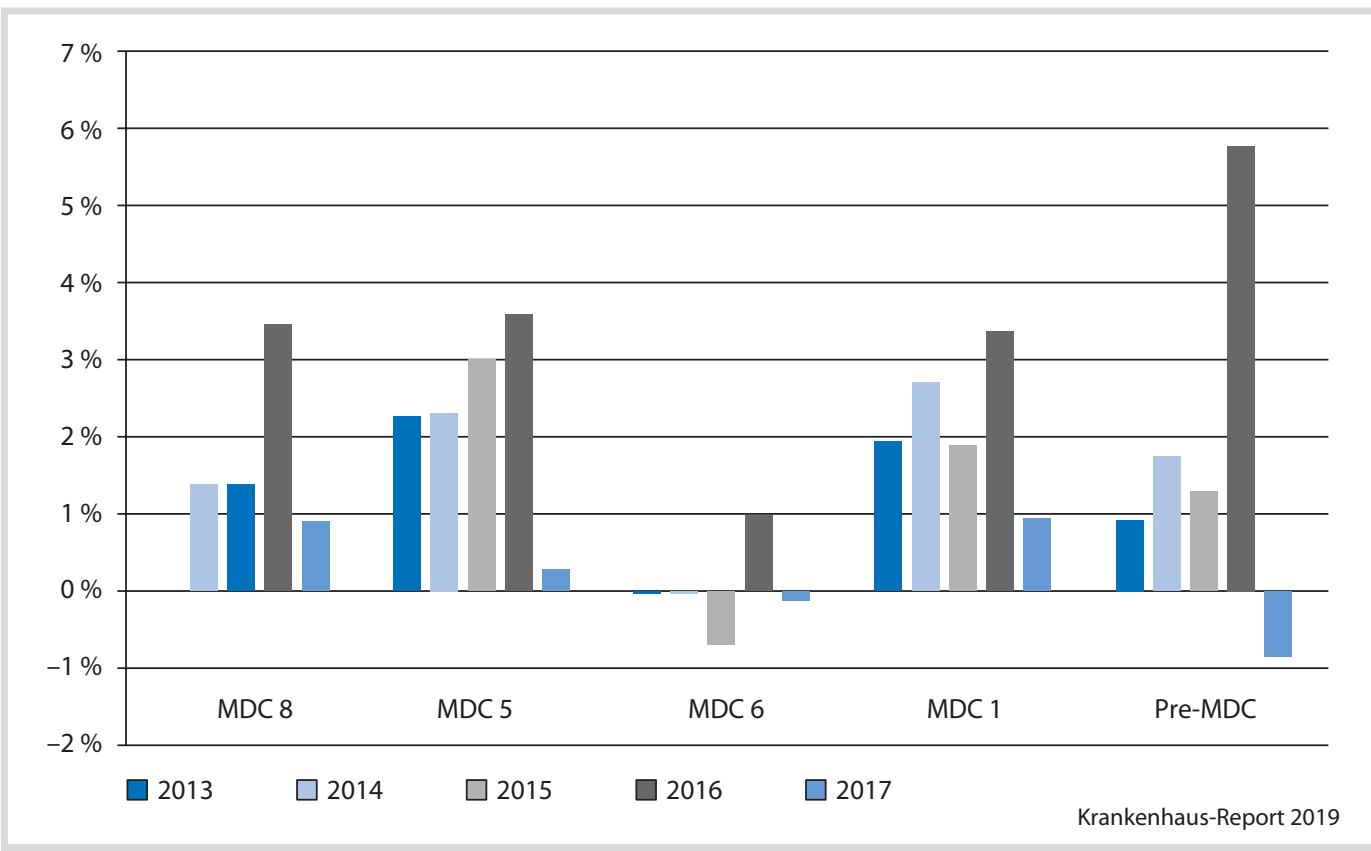

- Abb. 16.6 Prozentuale Veränderung des vereinbarten CM 2013-2017 der CM-stärksten MDCs 2017 ( $n=1.230$ Krankenhäuser)

1.468,5 Mio. Euro im Jahr 2017. Aufgrund der aus dem E2 ins E3.2 wechselnden Zusatzentgelte sinkt das E2-Budgetvolumen um 2,0 \%.

Wie bereits in den Vorjahren ist das ZE130 „Hochaufwendige Pflege von Erwachsenen“ 2017 mit 306,3 Mio. Euro das umsatzstärkste E2-Zusatzentgelt (•Tab. 16.5). Es weist darüber hinaus einen überdurchschnittlichen Anstieg von 15,3\% auf. An zweiter Stelle steht erneut das ZE148 „Gabe von Rituximab, intravenös“. Insgesamt machen beide Entgelte fast ein Drittel des gesamten Budgets für E2-Zusatzentgelte aus.

- Tab. 16.5 zerlegt die Budgetveränderung in Mengen-, Preis- und Struktureffekte wie zum Beispiel Verschiebungen zwischen Dosierungsklassen bei Medikamenten. So geht der starke Budgetanstieg beim ZE130 ausschließlich auf die Mengenkomponente zurück, während der Preis dämpfend wirkt. Eine Besonderheit stellt das ZE101 („Medikamente-freisetzende Koronarstents") dar. Bei diesem ist bereits seit Jahren ein deutlicher Budgetrückgang bei steigenden Mengen festzustellen (Mostert et al. 2018). Insgesamt hat sich die Reihenfolge der umsatzstärksten E2-Zusatzentgelte kaum verändert;
Ausnahme ist der Wegfall der beiden umsatzstarken Medikamente Caspofungin (früher ZE109) und Pemetrexed (früher ZE53), die in das E3.2 gewechselt sind.

Eine andere Betrachtungsweise bietet die Unterteilung der E2-Zusatzentgelte nach Segmenten, die so nicht im Katalog zu finden sind. Es handelt sich hierbei um die Zusatzentgelte für Dialyseverfahren, um Medikamentengaben sowie um die sonstigen Zusatzentgelte. Das letzte Segment ist heterogen und umfasst auch besondere Behandlungsverfahren, wie zum Beispiel ZE130 und ZE131 für die hochaufwendige Pflege.

Das Segment Dialyse und die sonstigen $\mathrm{Zu}$ satzentgelte weisen in diesem Jahr einen höheren Zuwachs auf als in den Vorjahren (Mostert et al. 2018). In diesen Bereichen wächst das Budget um 6,1 \% beziehungsweise 10,9\%. Bei den Medikamentengaben macht sich auch hier der Einfluss der aus dem E2 ausgegliederten Medikamente bemerkbar. Daher sinkt das Budget für dieses Segment um 14,4\% - insbesondere durch den hiermit verbundenen Mengenrückgang, aber auch infolge der durch Abgänge geprägten Strukturkomponente 


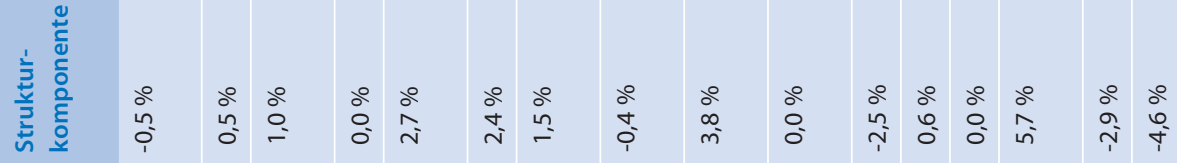

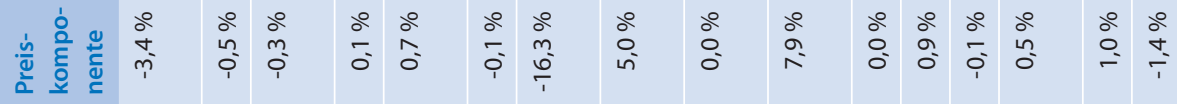

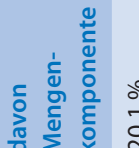

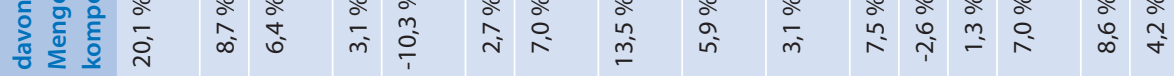

$\frac{1}{\frac{1}{2}} \frac{2}{\frac{1}{2}}$

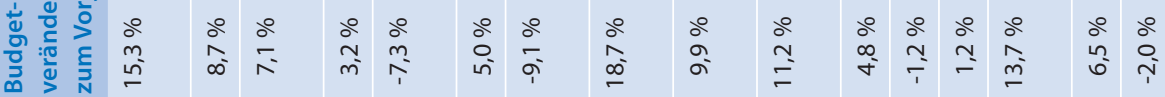

$\overline{\bar{d}}$

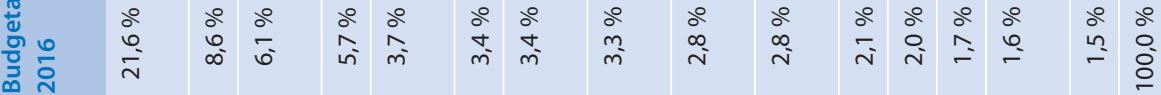

등 은

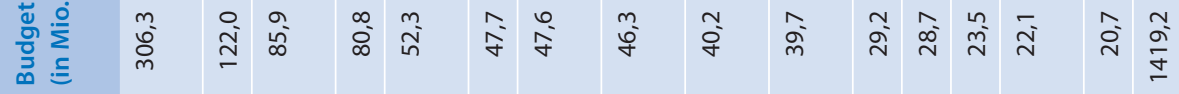

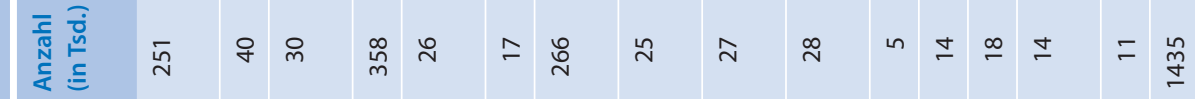

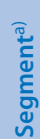

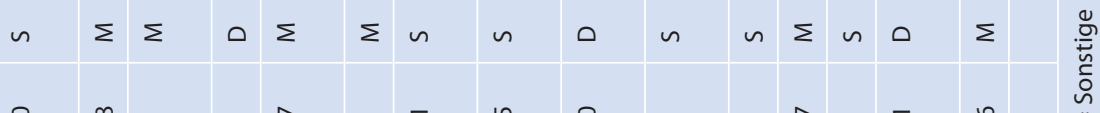

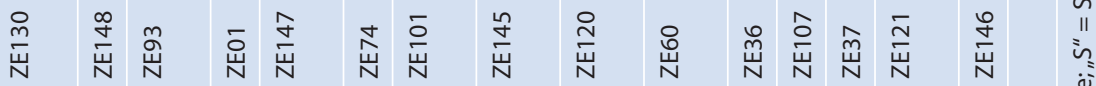

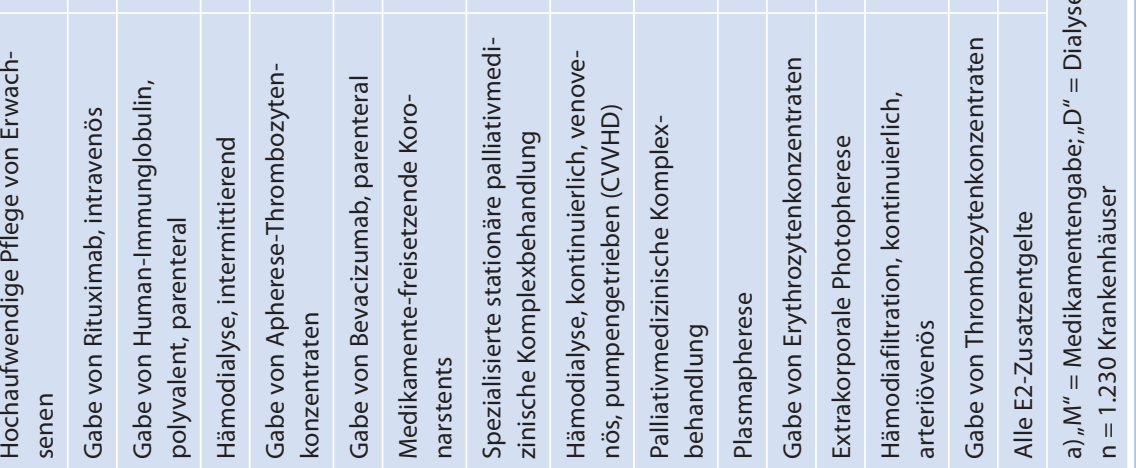


(•Tab. 16.6). Dieser Struktureffekt bedingt auch den Rückgang des Gesamtbudgets für das E2 um 2,0 \%, obwohl über alle E2-Zusatzentgelte betrachtet die Mengenkomponente positiv ist.

- Abb. 16.7 stellt die maßgeblichen Einflussfaktoren für die vereinbarten Budgetveränderungen bundeseinheitlicher Zusatzentgelte insgesamt nach der Methode der Komponentenzerlegung dar. ${ }^{13}$ Anders als bei der bisherigen Betrachtung wurden hierbei sowohl die $\mathrm{Zu}$ - als auch die Abgänge eliminiert. Der starke Einfluss der sechs aus dem E2 ausgegliederten Medikamentengaben fällt hier somit weg; es werden nur diejenigen Zusatzentgelte betrachtet, die in beiden Jahren im E2 enthalten waren. Unter diesem Blickwinkel zeigt sich, dass die Budgetentwicklung im E2-Zusatzentgeltebereich eindeutig von der Mengenentwicklung bestimmt wird. Die Preisentwicklung ist gegenläufig, wird jedoch von den strukturellen Veränderungen ziemlich genau kompensiert.

\subsection{Umsetzung der Verhandlungs- ergebnisse}

Der $\$ 4$ Absatz 2 Satz 1 KHEntgG sieht die Vorgabe einer leistungsorientierten Erlösbudgetermittlung vor, die sich nach den voraussichtlich zu erbringenden Leistungen richten soll. Bereits in den vergangenen Jahren war ein Trend hin zu späteren Umsetzungszeitpunkten zu beobachten, der für zunehmend unterjährige oder retrospektive Verhandlungen beziehungsweise Einigungen steht. ${ }^{14}$ Diese Entwicklung zeigt sich auch im Vergleich der Jahre 2016 und 2017 ${ }^{15}$ : Für das Budgetjahr 2016 wurden rund $54 \%$ des in Summe über alle LBFW vereinbarten CM-Volumens in den Einzelhausverhand-

$13 \mathrm{Zu}$ den methodischen Voraussetzungen der Anwendung der Komponentenzerlegung auf den Bereich der E2-Zusatzentgelte vergleiche Mostert et al. 2013.

14 Im Jahr 2011 lag die unterjährige Umsetzungsquote bei $72,5 \%$. Der Wert verringerte sich bis 2015 durchschnittlich um 3,7 \%punkte p. a.

15 Für die Analyse der Umsetzungszeitpunkte wurde von der Stichprobe der 1.230 Krankenhäuser, zu denen für 2016 und 2017 eine Budgetvereinbarung vorliegt, abgewichen. Stattdessen wurden alle im jeweiligen Budgetjahr vorliegenden Verhandlungsergebnisse einbezogen.

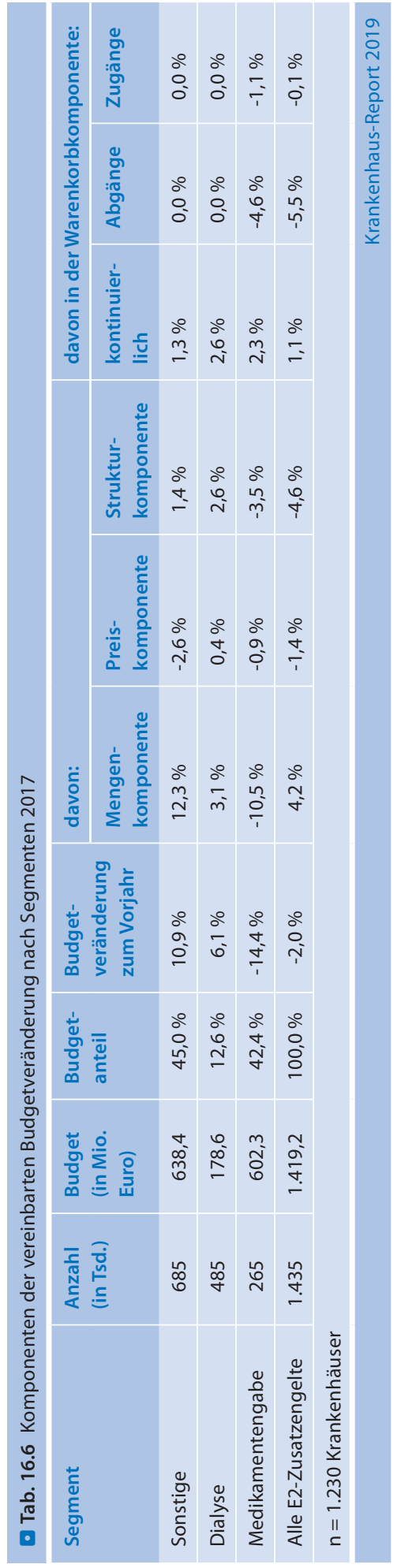




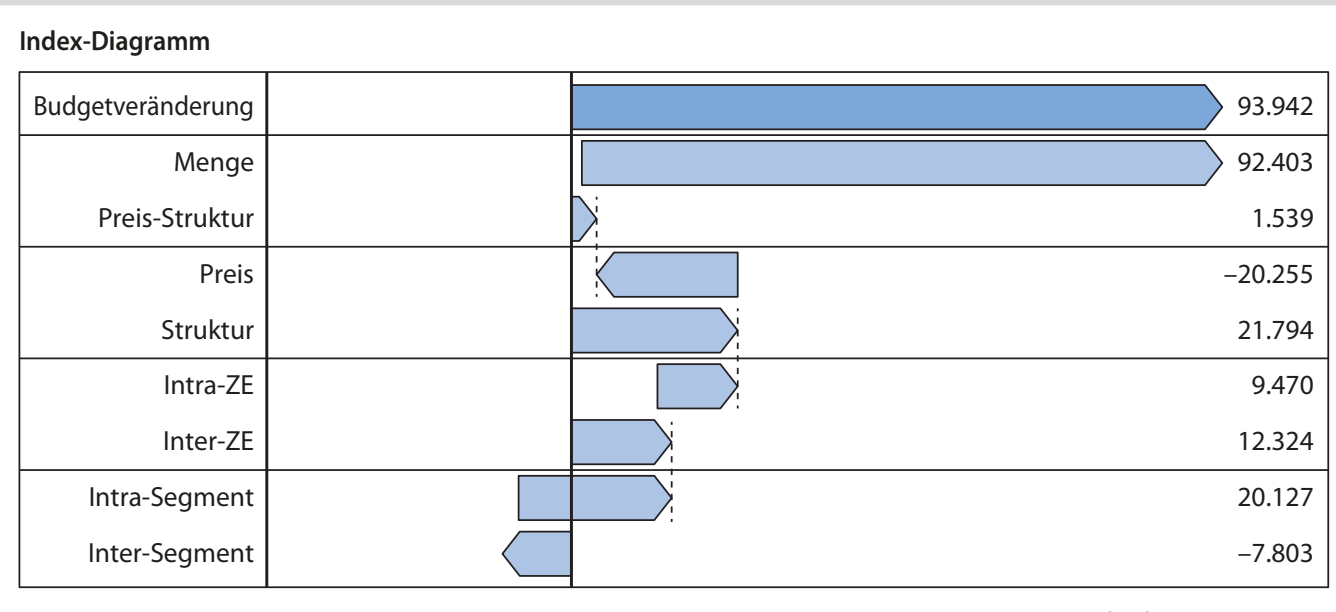

Krankenhaus-Report 2019

- Abb. 16.7 Komponenten der vereinbarten Budgetveränderung für bundeseinheitliche Zusatzentgelte (in Tsd. Euro), 2017 im Vergleich zum Vorjahr ( $n=1.230$ Krankenhäuser)

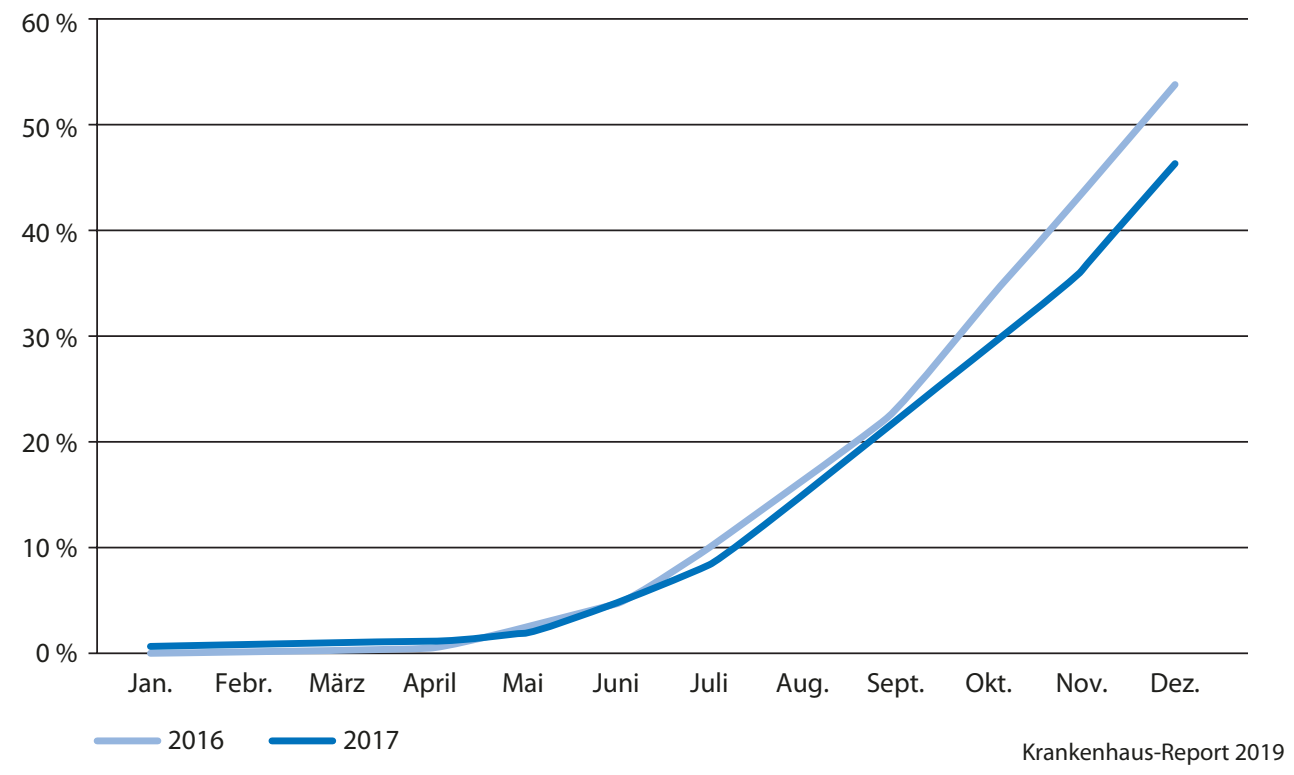

- Abb. 16.8 Umsetzungszeitpunkte 2016 und 2017 nach kumulierten CM-Anteilen

lungen unterjährig umgesetzt (• Abb. 16.8). 2017 wurden vor allem im letzten Quartal weniger Verhandlungsergebnisse genehmigt, sodass am Jahresende nur für $46 \%$ des CM-Volumens im LBFW Planungssicherheit bestand. Als Hauptursache für die verzögerte Umsetzung im Vergleich zum Vor- jahr kann der 2017 erstmals zu vereinbarende FDA gelten. Dieser neue Verhandlungstatbestand hat aufgrund seiner zahlreichen neuen, komplexen und vor allem auch streitbefangenen Regelungsdetails die Budgetverhandlungen von Krankenhäusern deutlich verzögert. 


\subsection{Zusammenfassung und Diskussion}

Die geänderten Rahmenbedingungen zur Budgetfindung aus dem Krankenhausstrukturgesetz finden im Jahr 2017 bereits in weiten Teilen Anwendung. Insbesondere die geänderten Regelungen im Kontext der Vereinbarung von Mehrmengen, wie die Einführung des Fixkostendegressionsabschlags, haben die Verhandlungsrunde 2017 nachhaltig geprägt, obwohl die Summe der vereinbarten Mehrleistungen in diesem Jahr so gering ausfiel wie lange nicht mehr. Dies äußerte sich daher weniger in monetären Sondereffekten als in deutlich späteren Verhandlungen und Umsetzungszeitpunkten, da viele Detailregelungen nicht nur neu und komplex, sondern auch sehr streitbefangen waren. Erstmals wurde weniger als die Hälfte des Gesamtbudgetvolumens innerhalb des Budgetjahres auch umgesetzt, mit den entsprechenden Konsequenzen für die Planungs- und Kalkulationssicherheit der Krankenhäuser und Kostenträger. In der Summe liegt die vereinbarte Preissteigerung mit 2,2\% nahe dem Mittelwert der letzten Jahre. Dies gilt allerdings auch deswegen, weil die anteilige Tariferhöhungs- rate im DRG-Bereich für 2017 in Höhe von 0,16 \% erst im Jahr 2018 budgeterhöhend wirken wird.

Deutlich abweichend zu den Vorjahren wurde allerdings die Mengenentwicklung vereinbart. Im Vorjahr lag die vereinbarte Mengensteigerung mit einem Plus von 2,7 \% so hoch wie seit Jahren nicht mehr. Im Jahr 2017 ist der Wert dagegen mit nur $0,9 \%$ rekordverdächtig niedrig ausgefallen. Dieser Einbruch in den Mehrmengenvereinbarungen zieht sich durch alle relevanten Hauptdiagnosegruppen des G-DRG-Systems (MDCs). Ob es sich hierbei um die Konsequenz aus massiven Vorholeffekten im Jahr 2016 handelt oder sich hier erste Anzeichen einer Trendumkehr bei der Mengenentwicklung im Krankenhausbereich zeigen, kann mit aktuellem Datenstand noch nicht beantwortet werden. Auch wäre die Deutung, dass diese moderate Mengenentwicklung auf die im gleichen Jahr geänderten Budgetmechaniken zurückzuführen ist, noch verfrüht.

Im Ergebnis sind die Budgets der untersuchten1.230 Krankenhäuser ausgleichsbereinigt um 3,0\% gestiegen, was einem Mittelzuwachs von knapp über 1,8 Mrd. Euro entspricht. Die Budgetsteigerungsrate weist damit den niedrigsten Wert seit 2011 auf.

\subsection{Anhang}

- Tab. 16.7 Zusatzentgelte 2016 und 2017

\begin{tabular}{|c|c|c|c|c|}
\hline ZE-Nr. & Segment & Bezeichnung & 2016 & 2017 \\
\hline ZE01 & D & Hämodialyse, intermittierend & $\mathrm{X}$ & $\mathrm{X}$ \\
\hline ZE02 & $\mathrm{D}$ & Hämodiafiltration, intermittierend & $\mathrm{X}$ & $\mathrm{X}$ \\
\hline ZE09 & $\mathrm{S}$ & Vollimplantierbare Medikamentenpumpe mit programmierbarem Tagesprofil & $\mathrm{X}$ & $\mathrm{x}$ \\
\hline ZE10 & S & Künstlicher Blasenschließmuskel, Eingriffe bei artifiziellem Harnblasensphinkter & $\mathrm{X}$ & $\mathrm{X}$ \\
\hline ZE11 & S & $\begin{array}{l}\text { Wirbelkörperersatz, Wirbelkörperersatz und komplexe Rekonstruktion der } \\
\text { Wirbelsäule }\end{array}$ & $x$ & $x$ \\
\hline ZE17 & M & Gabe von Gemcitabin, parenteral & $x$ & $x$ \\
\hline ZE19 & M & Gabe von Irinotecan, parenteral & $x$ & $x$ \\
\hline ZE30 & M & Gabe von Prothrombin-komplex, parenteral & $x$ & $x$ \\
\hline ZE36 & S & Plasmapherese & $x$ & $x$ \\
\hline ZE37 & $\mathrm{S}$ & Extrakorporale Photopherese & $x$ & $x$ \\
\hline ZE40 & M & Gabe von Filgrastim, parenteral & $x$ & $x$ \\
\hline ZE42 & M & Gabe von Lenograstim, parenteral & $x$ & $x$ \\
\hline ZE44 & M & Gabe von Topotecan, parenteral & $x$ & $x$ \\
\hline
\end{tabular}


- Tab. 16.7 (Fortsetzung)

\begin{tabular}{|c|c|c|c|c|}
\hline ZE-Nr. & Segment & Bezeichnung & 2016 & 2017 \\
\hline ZE47 & M & Gabe von Antithrombin III, parenteral & $\mathrm{x}$ & $x$ \\
\hline ZE48 & M & Gabe von Aldesleukin, parenteral & $\mathrm{x}$ & $x$ \\
\hline ZE49 & M & Gabe von Bortezomib, parenteral & $x$ & $x$ \\
\hline ZE50 & M & Gabe von Cetuximab, parenteral & $\mathrm{X}$ & $x$ \\
\hline ZE51 & M & $\begin{array}{l}\text { Gabe von Human-Immunglobulin, spezifisch gegen Hepatitis-B-surface- } \\
\text { Antigen, parenteral }\end{array}$ & $x$ & $x$ \\
\hline ZE52 & M & Gabe von Liposomalem Doxorubicin, parenteral & $\mathrm{x}$ & $x$ \\
\hline ZE53 & M & Gabe von Pemetrexed, parenteral & $x$ & \\
\hline ZE56 & $\mathrm{S}$ & Vollimplantierbare Medikamentenpumpe mit konstanter Flussrate & $\mathrm{x}$ & $x$ \\
\hline ZE58 & $\mathrm{S}$ & Hydraulische Penisprothesen, andere Operationen am Penis & $x$ & $x$ \\
\hline ZE60 & $\mathrm{S}$ & Palliativmedizinische Komplexbehandlung & $x$ & $x$ \\
\hline ZE61 & $\mathrm{S}$ & LDL-Apherese & $\mathrm{X}$ & $x$ \\
\hline ZE62 & $\mathrm{D}$ & Hämofiltration, intermittierend & $x$ & $\mathrm{x}$ \\
\hline ZE63 & M & Gabe von Paclitaxel, parenteral & $\mathrm{x}$ & $\mathrm{x}$ \\
\hline ZE64 & M & $\begin{array}{l}\text { Gabe von Human-Immunglobulin, spezifisch gegen Zytomegalie-Virus, } \\
\text { parenteral }\end{array}$ & $\mathrm{x}$ & $\mathrm{x}$ \\
\hline ZE66 & M & Gabe von Adalimumab, parenteral & $x$ & $\mathrm{x}$ \\
\hline ZE67 & M & $\begin{array}{l}\text { Gabe von Human-Immunglobulin, spezifisch gegen Varicella-Zoster-Virus, } \\
\text { parenteral }\end{array}$ & $x$ & $x$ \\
\hline ZE68 & M & Gabe von Infliximab, parenteral & $\mathrm{x}$ & $\mathrm{x}$ \\
\hline ZE70 & M & Gabe von C1-Esteraseinhibitor, parenteral & $\mathrm{x}$ & $\mathrm{x}$ \\
\hline ZE71 & M & Gabe von Pegfilgrastim, parenteral & $\mathrm{x}$ & $\mathrm{x}$ \\
\hline ZE72 & M & Gabe von Pegyliertem liposomalen Doxorubicin, parenteral & $x$ & $\mathrm{x}$ \\
\hline ZE74 & M & Gabe von Bevacizumab, parenteral & $x$ & $x$ \\
\hline ZE75 & M & Gabe von Liposomalem Cytarabin, intrathekal & $x$ & $x$ \\
\hline ZE76 & M & Gabe von Etanercept, parenteral & $x$ & \\
\hline ZE78 & M & Gabe von Temozolomid, oral & $x$ & $x$ \\
\hline ZE79 & M & Gabe von Busulfan, parenteral & $x$ & $x$ \\
\hline ZE80 & M & Gabe von Docetaxel, parenteral & $x$ & $x$ \\
\hline ZE92 & M & Gabe von Imatinib, oral & $x$ & \\
\hline ZE93 & M & Gabe von Human-Immun-globulin, polyvalent, parenteral & $x$ & $x$ \\
\hline ZE95 & M & Gabe von Palifermin, parenteral & $x$ & $x$ \\
\hline ZE96 & M & Gabe von Carmustin-Implantaten, intrathekal & $x$ & $x$ \\
\hline ZE97 & M & Gabe von Natalizumab, parenteral & $x$ & $x$ \\
\hline ZE98 & M & Gabe von Palivizumab, parenteral & $x$ & $x$ \\
\hline ZE100 & $\mathrm{S}$ & $\begin{array}{l}\text { Implantation eines endobronchialen Klappensystems, andere Operationen } \\
\text { an Lunge und Bronchien }\end{array}$ & $x$ & $x$ \\
\hline ZE101 & $\mathrm{S}$ & Medikamente-freisetzende Koronarstents & $x$ & $x$ \\
\hline ZE105 & $\mathrm{S}$ & $\begin{array}{l}\text { Selektive Embolisation mit Metallspiralen (Coils) an Kopf, Hals (intra- und extra- } \\
\text { kraniell) und spinalen Gefäßen oder mit großlumigem Gefäßverschlusskörper }\end{array}$ & $x$ & $x$ \\
\hline
\end{tabular}


- Tab. 16.7 (Fortsetzung)

\begin{tabular}{|c|c|c|c|c|}
\hline ZE-Nr. & Segment & Bezeichnung & 2016 & 2017 \\
\hline ZE106 & S & Selektive Embolisation mit Metallspiralen (Coils), andere Lokalisaion & $x$ & $\mathrm{x}$ \\
\hline ZE107 & M & Gabe von Erythrozytenkonzentraten & $\mathrm{X}$ & $\mathrm{x}$ \\
\hline ZE108 & M & Gabe von patientenbezogenen Thrombozytenkonzentraten & $\mathrm{X}$ & $\mathrm{x}$ \\
\hline ZE109 & M & Gabe von Caspofungin, parenteral & $\mathrm{X}$ & \\
\hline ZE110 & M & Gabe von Liposomalem Amphotericin B, parenteral & $\mathrm{X}$ & $\mathrm{X}$ \\
\hline ZE111 & M & Gabe von Voriconazol, oral & $x$ & \\
\hline ZE112 & M & Gabe von Voriconazol, parenteral & $x$ & \\
\hline ZE113 & M & Gabe von Itraconazol, parenteral & $x$ & $x$ \\
\hline ZE115 & M & Gabe von Anidulafungin, parenteral & $x$ & $x$ \\
\hline ZE116 & M & Gabe von Panitumumab, parenteral & $x$ & $x$ \\
\hline ZE117 & M & Gabe von Trabectedin, parenteral & $x$ & $x$ \\
\hline ZE119 & $\mathrm{D}$ & Hämofiltration, kontinuierlich & $\mathrm{X}$ & $X$ \\
\hline ZE120 & $\mathrm{D}$ & Hämodialyse, kontinuierlich, venovenös, pumpengetrieben (CVVHD) & $\mathrm{x}$ & $\mathrm{x}$ \\
\hline ZE121 & $\mathrm{D}$ & Hämodiafiltration, kontinuierlich & $\mathrm{X}$ & $\mathrm{x}$ \\
\hline ZE122 & $\mathrm{D}$ & Peritonealdialyse, intermittierend, maschinell unterstützt (IPD) & $\mathrm{X}$ & $\mathrm{X}$ \\
\hline ZE123 & $\mathrm{D}$ & Peritonealdialyse, kontinuierlich, nicht maschinell unterstützt (CAPD) & $x$ & $x$ \\
\hline ZE124 & M & Gabe von Azacytidin, parenteral & $\mathrm{x}$ & $\mathrm{x}$ \\
\hline ZE125 & $\mathrm{S}$ & $\begin{array}{l}\text { Implantation oder Wechsel eines interspinösen Spreizers, andere Operati- } \\
\text { onen an der Wirbelsäule }\end{array}$ & $\mathrm{x}$ & $\mathrm{x}$ \\
\hline ZE126 & S & Autogene/autologe matrixinduzierte Chondrozytentransplantation & $\mathrm{x}$ & $\mathrm{x}$ \\
\hline ZE128 & M & Gabe von Micafungin, parenteral & $\mathrm{x}$ & $x$ \\
\hline ZE130 & $\mathrm{S}$ & Hochaufwendige Pflege von Erwachsenen & $\mathrm{x}$ & $\mathrm{x}$ \\
\hline ZE131 & $\mathrm{S}$ & Hochaufwendige Pflege von Kleinkindern oder von Kindern und Jugendlichen & $\mathrm{X}$ & $\mathrm{x}$ \\
\hline ZE132 & $\mathrm{S}$ & Implantation eines Wachstumsstents & $\mathrm{x}$ & $\mathrm{x}$ \\
\hline ZE133 & $\mathrm{S}$ & $\begin{array}{l}\text { Perkutan transluminale Fremdkörperentfernung und Thrombektiomie an } \\
\text { intrakraniellen Gefäßen unter Verwendung eines Mikrodrahtretriever-Systems }\end{array}$ & $x$ & $\mathrm{x}$ \\
\hline ZE134 & $\mathrm{S}$ & Verschiedene Harnkontinenztherapien & $x$ & $x$ \\
\hline ZE135 & M & Gabe von Vinflunin, parenteral & $x$ & $x$ \\
\hline ZE136 & $\mathrm{S}$ & Medikamente-freisetzende Ballons an Koronargefäßen & $\mathrm{x}$ & $\mathrm{x}$ \\
\hline ZE137 & $\mathrm{S}$ & Medikamente-freisetzende Ballons an anderen Gefäßen & $\mathrm{x}$ & $x$ \\
\hline ZE138 & $\mathrm{S}$ & $\begin{array}{l}\text { Neurostimulatoren zur Rückenmarkstimulation oder zur Stimulation des } \\
\text { peripheren Nervensystems, Einkanalsystem, mit Sondenimplantation }\end{array}$ & $x$ & $\mathrm{x}$ \\
\hline ZE139 & $\mathrm{S}$ & $\begin{array}{l}\text { Neurostimulatoren zur Rückenmarkstimulation oder zur Stimulation des } \\
\text { peripheren Nervensystems, Einkanalsystem, ohne Sondenimplantation }\end{array}$ & $x$ & $x$ \\
\hline ZE140 & $\mathrm{S}$ & $\begin{array}{l}\text { Neurostimulatoren zur Rückenmarkstimulation oder zur Stimulation des } \\
\text { peripheren Nervensystems, Mehrkanalsystem, nicht wiederaufladbar, mit } \\
\text { Sondenimplantation }\end{array}$ & $x$ & $\mathrm{x}$ \\
\hline ZE141 & $\mathrm{S}$ & $\begin{array}{l}\text { Neurostimulatoren zur Rückenmarkstimulation oder zur Stimulation des } \\
\text { peripheren Nervensystems, Mehrkanalsystem, nicht wiederaufladbar, ohne } \\
\text { Sondenimplantation }\end{array}$ & $\mathrm{x}$ & $x$ \\
\hline ZE142 & M & Gabe von Clofarabin, parenteral & $x$ & $x$ \\
\hline
\end{tabular}


- Tab. 16.7 (Fortsetzung)

\begin{tabular}{|c|c|c|c|c|}
\hline ZE-Nr. & Segment & Bezeichnung & 2016 & 2017 \\
\hline ZE143 & M & Gabe von Plerixafor, parenteral & $\mathrm{X}$ & $\mathrm{X}$ \\
\hline ZE144 & M & Gabe von Romiplostim, parenteral & $\mathrm{X}$ & $\mathrm{X}$ \\
\hline ZE145 & S & Spezialisierte stationäre palliativmedizinische Komplexbehandlung & $\mathrm{X}$ & $\mathrm{X}$ \\
\hline ZE146 & M & Gabe von Thrombozytenkonzentraten & $\mathrm{X}$ & $\mathrm{X}$ \\
\hline ZE147 & M & Gabe von Apherese-Thrombozytenkonzentrat & $x$ & $\mathrm{x}$ \\
\hline ZE148 & M & Gabe von Rituximab, intravenös & $x$ & $x$ \\
\hline ZE149 & M & Gabe von Trastuzumab, intravenös & $\mathrm{x}$ & $\mathrm{X}$ \\
\hline ZE150 & M & Gabe von Posaconazol, oral & $\mathrm{X}$ & $\mathrm{X}$ \\
\hline ZE151 & M & Gabe von Abatacept, intravenös & $X$ & $X$ \\
\hline ZE152 & $\mathrm{S}$ & $\begin{array}{l}\text { Perkutan-transluminale Fremdkörperentfernung und Thrombektomie an } \\
\text { intrakraniellen Gefäßen unter Verwendung eines Stentretriever-Systems }\end{array}$ & $\mathrm{x}$ & $\mathrm{x}$ \\
\hline ZE153 & $S$ & Zügeloperation mit alloplastischem Material, adjustierbar & $\mathrm{X}$ & $\mathrm{X}$ \\
\hline ZE154 & M & Gabe von Eculizumab, parenteral & $\mathrm{X}$ & $\mathrm{X}$ \\
\hline ZE155 & M & Gabe von Ofatumumab, parenteral & $\mathrm{x}$ & $\mathrm{x}$ \\
\hline ZE156 & M & Gabe von Decitabine, parenteral & $\mathrm{X}$ & $\mathrm{X}$ \\
\hline ZE157 & M & Gabe von Tocilizumab, intravenös & $\mathrm{X}$ & $x$ \\
\hline ZE158 & $\mathrm{S}$ & Vagusnervstimulationssysteme, mit Sondenimplantation & $\mathrm{X}$ & $\mathrm{x}$ \\
\hline ZE159 & $\mathrm{S}$ & Vagusnervstimulationssysteme, ohne Sondenimplantation & $x$ & $\mathrm{X}$ \\
\hline ZE160 & M & Gabe von Lipegfilgrastim, parenteral & & $\mathrm{X}$ \\
\hline ZE161 & $\mathrm{S}$ & Radiofrequenzablation Ösophagus & & $\mathrm{X}$ \\
\hline
\end{tabular}

\section{Literatur}

Friedrich J, Günster C (2006) Determinanten der CM-Entwicklung in Deutschland während der Einführung von DRGs (2002 bis 2004). In: Klauber J, Robra, B-P, Schellschmidt, H (Hrsg) Krankenhaus-Report 2005. Schattauer, Stuttgart, S 153-202

Friedrich J, Paschen K (2005) Schätzfehler bei der Überleitung von Leistungsdaten verringern - das WIdO-Verfahren der "vereinbarungsgewichteten Überleitung". f\&w 5(22): 464-468

Fürstenberg T, Laschat M, Zick K, Klein S, Gierling P, Noting HP, Schmidt T (2013) G-DRG-Begleitforschung gemäß § 17b Abs. 8 KHG. Endbericht des dritten Forschungszyklus (2008-2010). https://www.g-drg.de/Datenbrowser_und_ Begleitforschung/Begleitforschung_DRG/Endbericht_ zum_dritten_Zyklus_der_G-DRG-Begleitforschung. Zugegriffen: 26. Oktober 2018

InEK (2016) Abschlussbericht. Weiterentwicklung des G-DRG-Systems für das Jahr 2017. https://www.g-drg.de/
Aktuelles/Abschlussbericht_und_Reportbrowser_zur_ Weiterentwicklung_des_G-DRG-Systems_2017/(language)/ger-DE. Zugegriffen: 26. Oktober 2018

Mostert C, Leclerque G, Friedrich J (2013) Eckdaten der Leistungsentwicklung im Krankenhausmarkt 2011. In: Klauber J, Geraedts M, Friedrich J, Wasem, J (Hrsg) Krankenhaus-Report 2013. Schattauer, Stuttgart, S 21-46

Mostert C, Leclerque G, Friedrich J (2015) Die Krankenhausbudgets 2012 und 2013 im Vergleich. In: Klauber J, Geraedts M, Friedrich J, Wasem, J (Hrsg) KrankenhausReport 2015. Schattauer, Stuttgart, S 303-324

Mostert C, Leclerque G, Friedrich J (2018) Die Krankenhausbudgets 2015 und 2016 im Vergleich. In: Klauber J, Geraedts M, Friedrich J, Wasem, J (Hrsg) KrankenhausReport 2018. Schattauer, Stuttgart, S 317-339

Reichelt H (1988) Eine Methode der statistischen Komponentenzerlegung. WIdO-Materialien 31. Wissenschaftliches Institut der AOK, Bonn 
Open Access Dieses Kapitel wird unter der Creative Commons Namensnennung 4.0 International Lizenz(http://creativecommons. org/licenses/by/4.0/deed.de) veröffentlicht, welche die Nutzung, Vervielfältigung, Bearbeitung, Verbreitung und Wiedergabe in jeglichem Medium und Format erlaubt, sofern Sie den/die ursprünglichen Autor(en) und die Quelle ordnungsgemäß nennen, einen Link zur Creative Commons Lizenz beifügen und angeben, ob Änderungen vorgenommen wurden.

Die in diesem Kapitel enthaltenen Bilder und sonstiges Drittmaterial unterliegen ebenfalls der genannten Creative Commons Lizenz, sofern sich aus der Abbildungslegende nichts anderes ergibt. Sofern das betreffende Material nicht unter der genannten Creative Commons Lizenz steht und die betreffende Handlung nicht nach gesetzlichen Vorschriften erlaubt ist, ist für die oben aufgeführten Weiterverwendungen des Materials die Einwilligung des jeweiligen Rechteinhabers einzuholen. 